\title{
Statistical Learning Theory
}

\section{Olivier Bousquet}

Department of Empirical Inference

Max Planck Institute of Biological Cybernetics

olivier. bousquet@tuebingen.mpg. de

Machine Learning Summer School, August 2003 


\section{Roadmap (1)}

- Lecture 1: Introduction

- Part I: Binary Classification

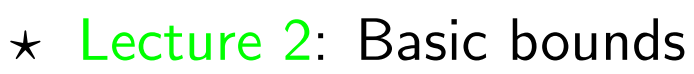

* Lecture 3: VC theory

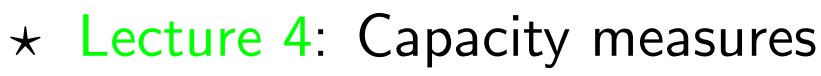

* Lecture 5: Advanced topics 


\section{Roadmap (2)}

- Part II: Real-Valued Classification

* Lecture 6: Margin and loss functions

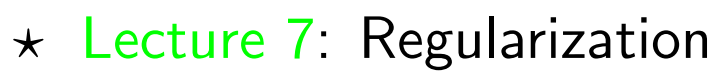

* Lecture 8: SVM

O. Bousquet - Statistical Learning Theory 


\section{Lecture 1}

\section{The Learning Problem}

- Context

- Formalization

- Approximation/Estimation trade-off

- Algorithms and Bounds

O. Bousquet - Statistical Learning Theory - Lecture 1 


\section{Learning and Inference}

The inductive inference process:

1. Observe a phenomenon

2. Construct a model of the phenomenon

3. Make predictions

$\Rightarrow$ This is more or less the definition of natural sciences!

$\Rightarrow$ The goal of Machine Learning is to automate this process

$\Rightarrow$ The goal of Learning Theory is to formalize it.

O. Bousquet - Statistical Learning Theory - Lecture 1 


\section{Pattern recognition}

We consider here the supervised learning framework for pattern recognition:

- Data consists of pairs (instance, label)

- Label is +1 or -1

- Algorithm constructs a function (instance $\rightarrow$ label)

- Goal: make few mistakes on future unseen instances

O. Bousquet - Statistical Learning Theory - Lecture 1 


\section{Approximation/Interpolation}

It is always possible to build a function that fits exactly the data.

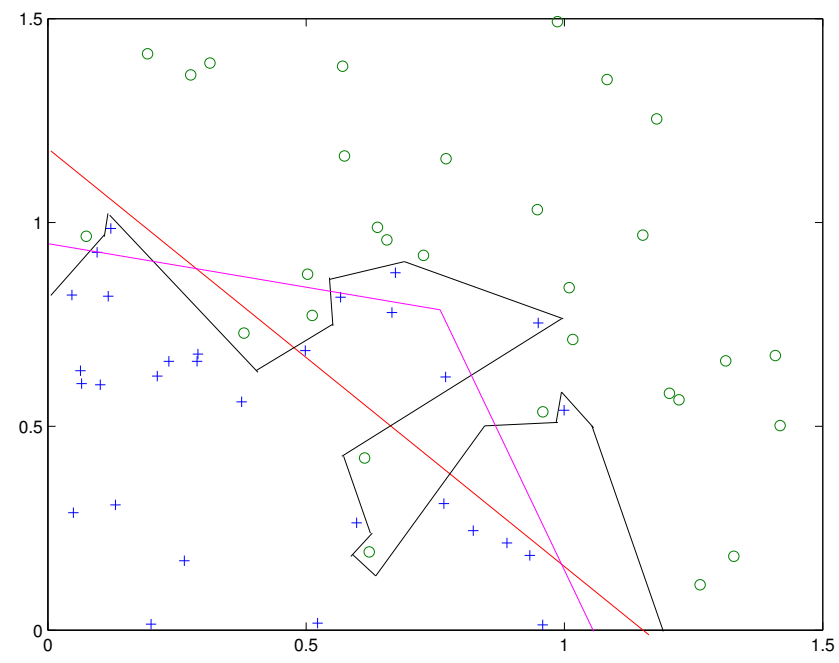

But is it reasonable?

O. Bousquet - Statistical Learning Theory - Lecture 1 


\section{Occam's Razor}

Idea: look for regularities in the observed phenomenon

These can ge generalized from the observed past to the future

$\Rightarrow$ choose the simplest consistent model

How to measure simplicity?

- Physics: number of constants

- Description length

- Number of parameters

O. Bousquet - Statistical Learning Theory - Lecture 1 


\section{No Free Lunch}

- No Free Lunch

$\star$ if there is no assumption on how the past is related to the future, prediction is impossible

$\star$ if there is no restriction on the possible phenomena, generalization is impossible

- We need to make assumptions

- Simplicity is not absolute

- Data will never replace knowledge

- Generalization = data + knowledge 


\section{Assumptions}

Two types of assumptions

- Future observations related to past ones

$\rightarrow$ Stationarity of the phenomenon

- Constraints on the phenomenon

$\rightarrow$ Notion of simplicity

O. Bousquet - Statistical Learning Theory - Lecture 1 


\section{Goals}

$\Rightarrow$ How can we make predictions from the past? what are the assumptions?

- Give a formal definition of learning, generalization, overfitting

- Characterize the performance of learning algorithms

- Design better algorithms

O. Bousquet - Statistical Learning Theory - Lecture 1 


\section{Probabilistic Model}

Relationship between past and future observations

$\Rightarrow$ Sampled independently from the same distribution

- Independence: each new observation yields maximum information

- Identical distribution: the observations give information about the underlying phenomenon (here a probability distribution)

O. Bousquet - Statistical Learning Theory - Lecture 1 


\section{Probabilistic Model}

We consider an input space $\mathcal{X}$ and output space $\mathcal{Y}$. Here: classification case $\mathcal{Y}=\{-1,1\}$.

Assumption: The pairs $(X, Y) \in \mathcal{X} \times \mathcal{Y}$ are distributed according to $P$ (unknown).

Data: We observe a sequence of $n$ i.i.d. pairs $\left(X_{i}, Y_{i}\right)$ sampled according to $P$.

Goal: construct a function $g: \mathcal{X} \rightarrow \mathcal{Y}$ which predicts $Y$ from $X$.

O. Bousquet - Statistical Learning Theory - Lecture 1 


\section{Probabilistic Model}

Criterion to choose our function:

Low probability of error $P(g(X) \neq Y)$.

Risk

$$
R(g)=P(g(X) \neq Y)=\mathbb{E}\left[1_{[g(X) \neq Y]}\right]
$$

- $P$ is unknown so that we cannot directly measure the risk

- Can only measure the agreement on the data

- Empirical Risk

$$
R_{n}(g)=\frac{1}{n} \sum_{i=1}^{n} 1_{\left[g\left(X_{i}\right) \neq Y_{i}\right]}
$$

O. Bousquet - Statistical Learning Theory - Lecture 1 


\section{Target function}

- $P$ can be decomposed as $P_{X} \times P(Y \mid X)$

- $\eta(x)=\mathbb{E}[Y \mid X=x]=2 \mathbb{P}[Y=1 \mid X=x]-1$ is the regression function

- $t(x)=\operatorname{sgn} \eta(x)$ is the target function

- in the deterministic case $Y=t(X)(\mathbb{P}[Y=1 \mid X] \in\{0,1\})$

- in general, $n(x)=\min (\mathbb{P}[Y=1 \mid X=x], 1-\mathbb{P}[Y=1 \mid X=x])=$ $(1-\eta(x)) / 2$ is the noise level 


\section{Assumptions about $P$}

Need assumptions about $P$.

Indeed, if $t(x)$ is totally chaotic, there is no possible generalization from finite data.

Assumptions can be

- Preference (e.g. a priori probability distribution on possible functions)

- Restriction (set of possible functions)

Treating lack of knowledge

- Bayesian approach: uniform distribution

- Learning Theory approach: worst case analysis

O. Bousquet - Statistical Learning Theory - Lecture 1 


\section{Approximation/Interpolation (again)}

How to trade-off knowledge and data?

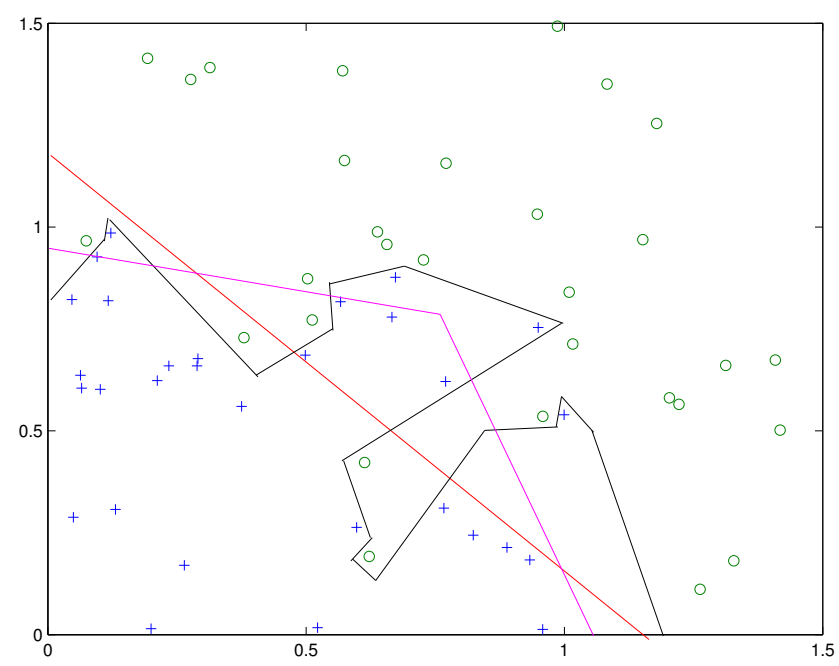

O. Bousquet - Statistical Learning Theory - Lecture 1 


\section{Overfitting/Underfitting}

The data can mislead you.

- Underfitting model too small to fit the data

- Overfitting artificially good agreement with the data

No way to detect them from the data! Need extra validation data.

O. Bousquet - Statistical Learning Theory - Lecture 1 


\section{Empirical Risk Minimization}

- Choose a model $\mathcal{G}$ (set of possible functions)

- Minimize the empirical risk in the model

$$
\min _{g \in \mathcal{G}} R_{n}(g)
$$

What if the Bayes classifier is not in the model ?

O. Bousquet - Statistical Learning Theory - Lecture 1 


\section{Approximation/Estimation}

- Bayes risk

$$
R^{*}=\inf _{g} R(g) .
$$

Best risk a deterministic function can have (risk of the target function, or Bayes classifier).

- Decomposition: $R\left(g^{*}\right)=\inf _{g \in \mathcal{G}} R(g)$

$$
R\left(g_{n}\right)-R^{*}=\underbrace{R(g)-R^{*}}_{\text {Approximation }}+\underbrace{R\left(g_{n}\right)-R\left(g^{*}\right)}_{\text {Estimation }}
$$

- Only the estimation error is random (i.e. depends on the data).

O. Bousquet - Statistical Learning Theory - Lecture 1 


\section{Structural Risk Minimization}

- Choose a collection of models $\left\{\mathcal{G}_{d}: d=1,2, \ldots\right\}$

- Minimize the empirical risk in each model

- Minimize the penalized empirical risk

$$
\min _{d} \min _{g \in \mathcal{G}_{d}} R_{n}(g)+\operatorname{pen}(d, n)
$$

$\operatorname{pen}(d, n)$ gives preference to models where estimation error is small $\operatorname{pen}(d, n)$ measures the size or capacity of the model 


\section{Regularization}

- Choose a large model $\mathcal{G}$ (possibly dense)

- Choose a regularizer $\|g\|$

- Minimize the regularized empirical risk

$$
\min _{g \in \mathcal{G}} R_{n}(g)+\lambda\|g\|^{2}
$$

- Choose an optimal trade-off $\lambda$ (regularization parameter).

Most methods can be thought of as regularization methods. 


\section{Bounds (1)}

A learning algorithm

- Takes as input the data $\left(X_{1}, Y_{1}\right), \ldots,\left(X_{n}, Y_{n}\right)$

- Produces a function $g_{n}$

Can we estimate the risk of $g_{n}$ ?

$\Rightarrow$ random quantity (depends on the data).

$\Rightarrow$ need probabilistic bounds

O. Bousquet - Statistical Learning Theory - Lecture 1 


\section{Bounds (2)}

- Error bounds

$$
R\left(g_{n}\right) \leq R_{n}\left(g_{n}\right)+B
$$

$\Rightarrow$ Estimation from an empirical quantity

- Relative error bounds

$\star$ Best in a class

$$
R\left(g_{n}\right) \leq R\left(g^{*}\right)+B
$$

$\star$ Bayes risk

$$
R\left(g_{n}\right) \leq R^{*}+B
$$

$\Rightarrow$ Theoretical guarantees

O. Bousquet - Statistical Learning Theory - Lecture 1 


\section{Lecture 2}

\section{Basic Bounds}

- Probability tools

- Relationship with empirical processes

- Law of large numbers

- Union bound

- Relative error bounds

O. Bousquet - Statistical Learning Theory - Lecture 2 


\section{Probability Tools (1)}

Basic facts

- Union: $\mathbb{P}[A$ or $B] \leq \mathbb{P}[A]+\mathbb{P}[B]$

- Inclusion: If $A \Rightarrow B$, then $\mathbb{P}[A] \leq \mathbb{P}[B]$.

- Inversion: If $\mathbb{P}[X \geq t] \leq F(t)$ then with probability at least $1-\delta$, $X \leq F^{-1}(\delta)$.

- Expectation: If $X \geq 0, \mathbb{E}[X]=\int_{0}^{\infty} \mathbb{P}[X \geq t] d t$.

O. Bousquet - Statistical Learning Theory - Lecture 2 


\section{Probability Tools (2)}

Basic inequalities

- Jensen: for $f$ convex, $f(\mathbb{E}[X]) \leq \mathbb{E}[f(X)]$

- Markov: If $X \geq 0$ then for all $t>0, \mathbb{P}[X \geq t] \leq \frac{\mathbb{E}[X]}{t}$

- Chebyshev: for $t>0, \mathbb{P}[|X-\mathbb{E}[X]| \geq t] \leq \frac{\operatorname{Var}[X]}{t^{2}}$

- Chernoff: for all $t \in \mathbb{R}, \mathbb{P}[X \geq t] \leq \inf _{\lambda \geq 0} \mathbb{E}\left[e^{\lambda(X-t)}\right]$

O. Bousquet - Statistical Learning Theory - Lecture 2 


\section{Error bounds}

Recall that we want to bound $R\left(g_{n}\right)=\mathbb{E}\left[1_{\left[g_{n}(X) \neq Y\right]}\right]$ where $g_{n}$ has been constructed from $\left(X_{1}, Y_{1}\right), \ldots,\left(X_{n}, Y_{n}\right)$.

- Cannot be observed ( $P$ is unknown)

- Random (depends on the data)

$\Rightarrow$ we want to bound

$$
\mathbb{P}\left[R\left(g_{n}\right)-R_{n}\left(g_{n}\right)>\varepsilon\right]
$$

O. Bousquet - Statistical Learning Theory - Lecture 2 


\section{Loss class}

For convenience, let $Z_{i}=\left(X_{i}, Y_{i}\right)$ and $Z=(X, Y)$. Given $\mathcal{G}$ define the loss class

$$
\mathcal{F}=\left\{f:(x, y) \mapsto 1_{[g(x) \neq y]}: g \in \mathcal{G}\right\}
$$

Denote $P f=\mathbb{E}[f(X, Y)]$ and $P_{n} f=\frac{1}{n} \sum_{i=1}^{n} f\left(X_{i}, Y_{i}\right)$

Quantity of interest:

$$
P f-P_{n} f
$$

We will go back and forth between $\mathcal{F}$ and $\mathcal{G}$ (bijection)

O. Bousquet - Statistical Learning Theory - Lecture 2 


\section{Empirical process}

Empirical process:

$$
\left\{P f-P_{n} f\right\}_{f \in \mathcal{F}}
$$

- Process $=$ collection of random variables (here indexed by functions in $\mathcal{F}$ )

- Empirical $=$ distribution of each random variable

$\Rightarrow$ Many techniques exist to control the supremum

$$
\sup _{f \in \mathcal{F}} P f-P_{n} f
$$

O. Bousquet - Statistical Learning Theory - Lecture 2 


\section{The Law of Large Numbers}

$$
R(g)-R_{n}(g)=\mathbb{E}[f(Z)]-\frac{1}{n} \sum_{i=1}^{n} f\left(Z_{i}\right)
$$

$\rightarrow$ difference between the expectation and the empirical average of the r.v. $f(Z)$

Law of large numbers

$$
\mathbb{P}\left[\lim _{n \rightarrow \infty} \frac{1}{n} \sum_{i=1}^{n} f\left(Z_{i}\right)-\mathbb{E}[f(Z)]=0\right]=1 .
$$

$\Rightarrow$ can we quantify it ?

O. Bousquet - Statistical Learning Theory - Lecture 2 


\section{Hoeffding's Inequality}

Quantitative version of law of large numbers.

Assumes bounded random variables

Theorem 1. Let $Z_{1}, \ldots, Z_{n}$ be $n$ i.i.d. random variables. If $f(Z) \in$ $[a, b]$. Then for all $\varepsilon>0$, we have

$$
\mathbb{P}\left[\left|\frac{1}{n} \sum_{i=1}^{n} f\left(Z_{i}\right)-\mathbb{E}[f(Z)]\right|>\varepsilon\right] \leq 2 \exp \left(-\frac{2 n \varepsilon^{2}}{(b-a)^{2}}\right) .
$$

$\Rightarrow$ Let's rewrite it to better understand

O. Bousquet - Statistical Learning Theory - Lecture 2 


\section{Hoeffding's Inequality}

Write

Then

$$
\delta=2 \exp \left(-\frac{2 n \varepsilon^{2}}{(b-a)^{2}}\right)
$$

or [Inversion] with probability at least $1-\delta$,

$$
\mathbb{P}\left[\left|P_{n} f-P f\right|>(b-a) \sqrt{\frac{\log \frac{2}{\delta}}{2 n}}\right] \leq \delta
$$

$$
\left|P_{n} f-P f\right| \leq(b-a) \sqrt{\frac{\log \frac{2}{\delta}}{2 n}}
$$

O. Bousquet - Statistical Learning Theory - Lecture 2 


\section{Hoeffding's inequality}

Let's apply to $f(Z)=1_{[g(X) \neq Y]}$.

For any $g$, and any $\delta>0$, with probability at least $1-\delta$

$$
R(g) \leq R_{n}(g)+\sqrt{\frac{\overline{\log \frac{2}{\delta}}}{2 n}}
$$

Notice that one has to consider a fixed function $f$ and the probability is with respect to the sampling of the data.

If the function depends on the data this does not apply!

O. Bousquet - Statistical Learning Theory - Lecture 2 


\section{Limitations}

- For each fixed function $f \in \mathcal{F}$, there is a set $S$ of samples for which $P f-P_{n} f \leq \sqrt{\frac{\log \frac{2}{\delta}}{2 n}}(\mathbb{P}[S] \geq 1-\delta)$

- They may be different for different functions

- The function chosen by the algorithm depends on the sample

$\Rightarrow$ For the observed sample, only some of the functions in $\mathcal{F}$ will satisfy this inequality!

O. Bousquet - Statistical Learning Theory - Lecture 2 


\section{Limitations}

What we need to bound is

$$
P f_{n}-P_{n} f_{n}
$$

where $f_{n}$ is the function chosen by the algorithm based on the data. For any fixed sample, there exists a function $f$ such that

$$
P f-P_{n} f=1
$$

Take the function which is $f\left(X_{i}\right)=Y_{i}$ on the data and $f(X)=-Y$ everywhere else.

This does not contradict Hoeffding but shows it is not enough

O. Bousquet - Statistical Learning Theory - Lecture 2 


\section{Limitations}

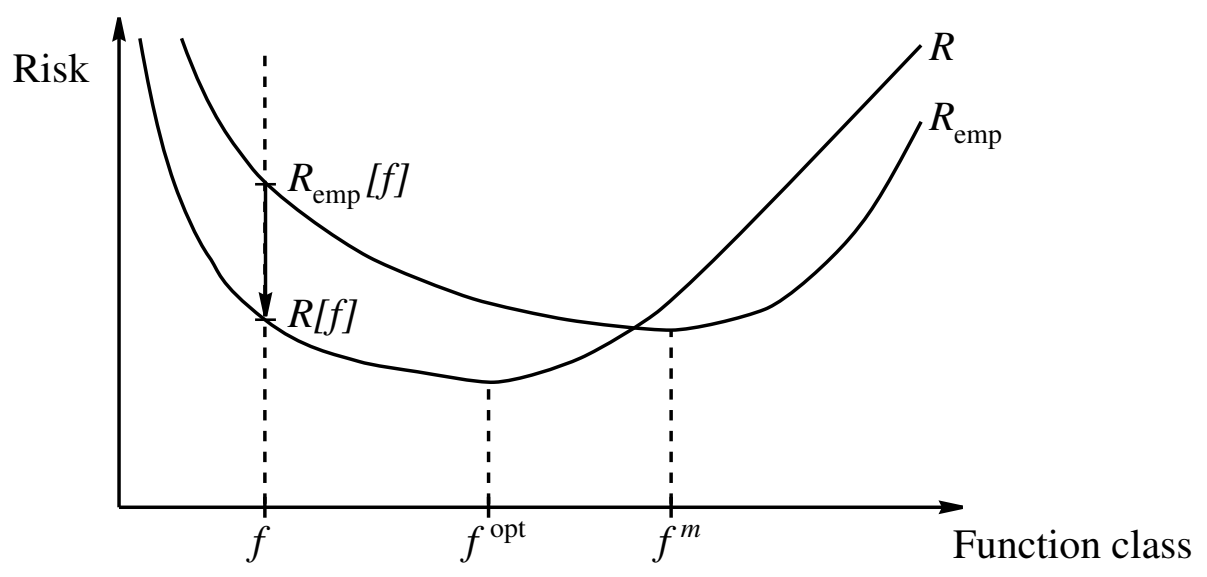

Hoeffding's inequality quantifies differences for a fixed function

O. Bousquet - Statistical Learning Theory - Lecture 2 


\section{Uniform Deviations}

Before seeing the data, we do not know which function the algorithm will choose.

The trick is to consider uniform deviations

$$
R\left(f_{n}\right)-R_{n}\left(f_{n}\right) \leq \sup _{f \in \mathcal{F}}\left(R(f)-R_{n}(f)\right)
$$

We need a bound which holds simultaneously for all functions in a class

O. Bousquet - Statistical Learning Theory - Lecture 2 


\section{Union Bound}

Consider two functions $f_{1}, f_{2}$ and define

$$
C_{i}=\left\{\left(x_{1}, y_{1}\right), \ldots,\left(x_{n}, y_{n}\right): P f_{i}-P_{n} f_{i}>\varepsilon\right\}
$$

From Hoeffding's inequality, for each $i$

$$
\mathbb{P}\left[C_{i}\right] \leq \delta
$$

We want to bound the probability of being 'bad' for $i=1$ or $i=2$

$$
\mathbb{P}\left[C_{1} \cup C_{2}\right] \leq \mathbb{P}\left[C_{1}\right]+\mathbb{P}\left[C_{2}\right]
$$

O. Bousquet - Statistical Learning Theory - Lecture 2 


\section{Finite Case}

More generally

$$
\mathbb{P}\left[C_{1} \cup \ldots \cup C_{N}\right] \leq \sum_{i=1}^{N} \mathbb{P}\left[C_{i}\right]
$$

We have

$$
\begin{aligned}
\mathbb{P} & {\left[\exists f \in\left\{f_{1}, \ldots, f_{N}\right\}: P f-P_{n} f>\varepsilon\right] } \\
& \leq \sum_{i=1}^{N} \mathbb{P}\left[P f_{i}-P_{n} f_{i}>\varepsilon\right] \\
& \leq N \exp \left(-2 n \varepsilon^{2}\right)
\end{aligned}
$$

O. Bousquet - Statistical Learning Theory - Lecture 2 


\section{Finite Case}

We obtain, for $\mathcal{G}=\left\{g_{1}, \ldots, g_{N}\right\}$, for all $\delta>0$

with probability at least $1-\delta$,

$$
\forall g \in \mathcal{G}, \quad R(g) \leq R_{n}(g)+\sqrt{\frac{\log N+\log \frac{1}{\delta}}{2 m}}
$$

This is a generalization bound!

Coding interpretation

$\log N$ is the number of bits to specify a function in $\mathcal{F}$

O. Bousquet - Statistical Learning Theory - Lecture 2 


\section{Approximation/Estimation}

Let

$$
g^{*}=\arg \min _{g \in \mathcal{G}} R(g)
$$

If $g_{n}$ minimizes the empirical risk in $\mathcal{G}$,

$$
R_{n}\left(g^{*}\right)-R_{n}\left(g_{n}\right) \geq 0
$$

Thus

$$
\begin{aligned}
R\left(g_{n}\right) & =R\left(g_{n}\right)-R\left(g^{*}\right)+R\left(g^{*}\right) \\
& \leq R_{n}\left(g^{*}\right)-R_{n}\left(g_{n}\right)+R\left(g_{n}\right)-R\left(g^{*}\right)+R\left(g^{*}\right) \\
& \leq 2 \sup _{g \in \mathcal{G}}\left|R(g)-R_{n}(g)\right|+R\left(g^{*}\right)
\end{aligned}
$$

O. Bousquet - Statistical Learning Theory - Lecture 2 


\section{Approximation/Estimation}

We obtain with probability at least $1-\delta$

$$
R\left(g_{n}\right) \leq R\left(g^{*}\right)+2 \sqrt{\frac{\log N+\log \frac{2}{\delta}}{2 m}}
$$

The first term decreases if $N$ increases

The second term increases

The size of $\mathcal{G}$ controls the trade-off

O. Bousquet - Statistical Learning Theory - Lecture 2 


\section{Summary (1)}

- Inference requires assumptions

- Data sampled i.i.d. from $P$

- Restrict the possible functions to $\mathcal{G}$

- Choose a sequence of models $\mathcal{G}_{m}$ to have more flexibility/control 


\section{Summary (2)}

- Bounds are valid w.r.t. repeated sampling

- For a fixed function $g$, for most of the samples

$$
R(g)-R_{n}(g) \approx 1 / \sqrt{n}
$$

- For most of the samples if $|\mathcal{G}|=N$

$$
\sup _{g \in \mathcal{G}} R(g)-R_{n}(g) \approx \sqrt{\log N / n}
$$

$\Rightarrow$ Extra variability because the chosen $g_{n}$ changes with the data 


\section{Improvements}

We obtained

$$
\sup _{g \in \mathcal{G}} R(g)-R_{n}(g) \leq \sqrt{\frac{\log N+\log \frac{2}{\delta}}{2 n}}
$$

To be improved

- Hoeffding only uses boundedness, not the variance

- Union bound as bad as if independent

- Supremum is not what the algorithm chooses.

Next we improve the union bound and extend it to the infinite case 


\section{Refined union bound (1)}

For each $f \in \mathcal{F}$,

$$
\begin{gathered}
\mathbb{P}\left[P f-P_{n} f>\sqrt{\left.\frac{\log \frac{1}{\delta(f)}}{2 n}\right]} \leq \delta(f)\right. \\
\mathbb{P}\left[\exists f \in \mathcal{F}: P f-P_{n} f>\sqrt{\frac{\log \frac{1}{\delta(f)}}{2 n}}\right] \leq \sum_{f \in \mathcal{F}} \delta(f)
\end{gathered}
$$

Choose $\delta(f)=\delta p(f)$ with $\sum_{f \in \mathcal{F}} p(f)=1$

O. Bousquet - Statistical Learning Theory - Lecture 2 


\section{Refined union bound (2)}

With probability at least $1-\delta$,

$$
\forall f \in \mathcal{F}, P f \leq P_{n} f+\sqrt{\frac{\log \frac{1}{p(f)}+\log \frac{1}{\delta}}{2 n}}
$$

- Applies to countably infinite $\mathcal{F}$

- Can put knowledge about the algorithm into $p(f)$

- But $p$ chosen before seeing the data 


\section{Refined union bound (3)}

- Good $p$ means good bound. The bound can be improved if you know ahead of time the chosen function (knowledge improves the bound)

- In the infinite case, how to choose the $p$ (since it implies an ordering)

- The trick is to look at $\mathcal{F}$ through the data

O. Bousquet - Statistical Learning Theory - Lecture 2 


\section{Lecture 3}

\section{Infinite Case: Vapnik-Chervonenkis Theory}

- Growth function

- Vapnik-Chervonenkis dimension

- Proof of the VC bound

- VC entropy

- SRM

O. Bousquet - Statistical Learning Theory - Lecture 3 


\section{Infinite Case}

Measure of the size of an infinite class?

- Consider

$$
\mathcal{F}_{z_{1}, \ldots, z_{n}}=\left\{\left(f\left(z_{1}\right), \ldots, f\left(z_{n}\right)\right): f \in \mathcal{F}\right\}
$$

The size of this set is the number of possible ways in which the data $\left(z_{1}, \ldots, z_{n}\right)$ can be classified.

- Growth function

$$
S_{\mathcal{F}}(n)=\sup _{\left(z_{1}, \ldots, z_{n}\right)}\left|\mathcal{F}_{z_{1}, \ldots, z_{n}}\right|
$$

- Note that $S_{\mathcal{F}}(n)=S_{\mathcal{G}}(n)$

O. Bousquet - Statistical Learning Theory - Lecture 3 


\section{Infinite Case}

- Result (Vapnik-Chervonenkis) With probability at least $1-\delta$

$$
\forall g \in \mathcal{G}, \quad R(g) \leq R_{n}(g)+\sqrt{\frac{\log S_{\mathcal{G}}(2 n)+\log \frac{4}{\delta}}{8 n}}
$$

- Always better than $N$ in the finite case

- How to compute $S_{\mathcal{G}}(n)$ in general ?

$\Rightarrow$ use VC dimension

O. Bousquet - Statistical Learning Theory - Lecture 3 


\section{VC Dimension}

Notice that since $g \in\{-1,1\}, S_{\mathcal{G}}(n) \leq 2^{n}$

If $S_{\mathcal{G}}(n)=2^{n}$, the class of functions can generate any classification on $n$ points (shattering)

Definition 2. The VC-dimension of $\mathcal{G}$ is the largest $n$ such that

$$
S_{\mathcal{G}}(n)=2^{n}
$$

O. Bousquet - Statistical Learning Theory - Lecture 3 


\section{VC Dimension}

\section{Hyperplanes}

In $\mathbb{R}^{d}, V C$ (hyperplanes $)=d+1$

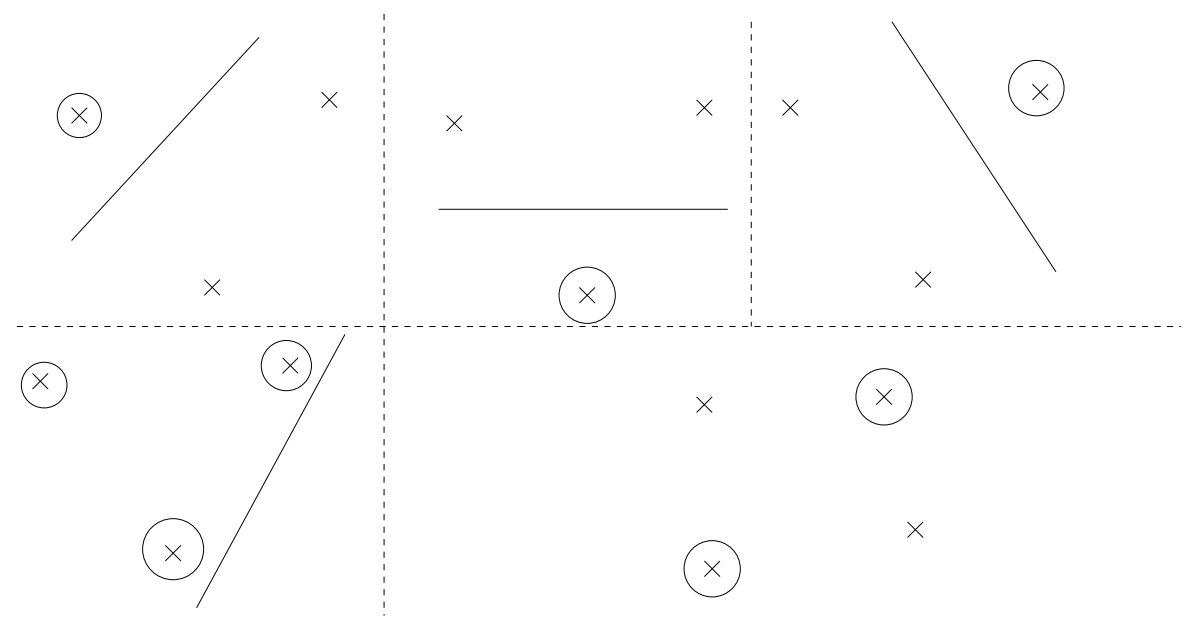

O. Bousquet - Statistical Learning Theory - Lecture 3 


\section{VC Dimension}

\section{Number of Parameters}

Is VC dimension equal to number of parameters?

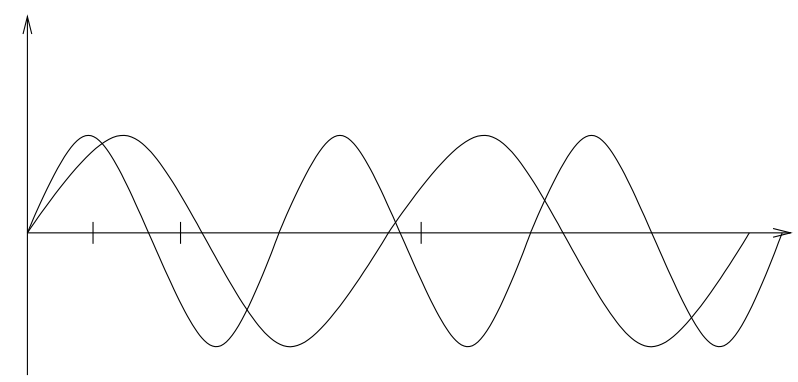

- One parameter

$$
\{\operatorname{sgn}(\sin (t x)): t \in \mathbb{R}\}
$$

- Infinite VC dimension!

O. Bousquet - Statistical Learning Theory - Lecture 3 


\section{VC Dimension}

- We want to know $S_{\mathcal{G}}(n)$ but we only know $S_{\mathcal{G}}(n)=2^{n}$ for $n \leq h$

What happens for $n \geq h$ ?

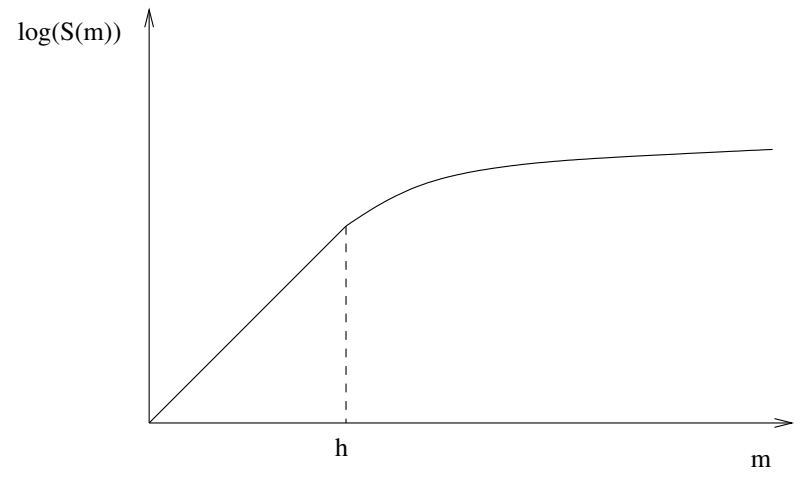

O. Bousquet - Statistical Learning Theory - Lecture 3 


\section{Vapnik-Chervonenkis-Sauer-Shelah Lemma}

Lemma 3. Let $\mathcal{G}$ be a class of functions with finite VC-dimension $h$. Then for all $n \in \mathbb{N}$,

$$
S_{\mathcal{G}}(n) \leq \sum_{i=0}^{h}\left(\begin{array}{l}
n \\
i
\end{array}\right)
$$

and for all $n \geq h$,

$$
S_{\mathcal{G}}(n) \leq\left(\frac{e n}{h}\right)^{h}
$$

$\Rightarrow$ phase transition

O. Bousquet - Statistical Learning Theory - Lecture 3 


\section{VC Bound}

Let $\mathcal{G}$ be a class with VC dimension $h$.

With probability at least $1-\delta$

$$
\forall g \in \mathcal{G}, \quad R(g) \leq R_{n}(g)+\sqrt{\frac{h \log \frac{2 e n}{h}+\log \frac{4}{\delta}}{8 n}}
$$

So the error is of order

$$
\sqrt{\frac{h \log n}{n}}
$$

O. Bousquet - Statistical Learning Theory - Lecture 3 


\section{Interpretation}

VC dimension: measure of effective dimension

- Depends on geometry of the class

- Gives a natural definition of simplicity (by quantifying the potential overfitting)

- Not related to the number of parameters

- Finiteness guarantees learnability under any distribution

O. Bousquet - Statistical Learning Theory - Lecture 3 


\section{Symmetrization (lemma)}

Key ingredient in VC bounds: Symmetrization

Let $Z_{1}^{\prime}, \ldots, Z_{n}^{\prime}$ an independent (ghost) sample and $P_{n}^{\prime}$ the corresponding empirical measure.

Lemma 4. For any $t>0$, such that $n t^{2} \geq 2$,

$$
\mathbb{P}\left[\sup _{f \in \mathcal{F}}\left(P-P_{n}\right) f \geq t\right] \leq 2 \mathbb{P}\left[\sup _{f \in \mathcal{F}}\left(P_{n}^{\prime}-P_{n}\right) f \geq t / 2\right]
$$

O. Bousquet - Statistical Learning Theory - Lecture 3 


\section{Symmetrization (proof - 1)}

$f_{n}$ the function achieving the supremum (depends on $Z_{1}, \ldots, Z_{n}$ )

$$
\begin{aligned}
1_{\left[\left(P-P_{n}\right) f_{n}>t\right]} 1_{\left[\left(P-P_{n}^{\prime}\right) f_{n}<t / 2\right]} & =1_{\left[\left(P-P_{n}\right) f_{n}>t \wedge\left(P-P_{n}^{\prime}\right) f_{n}<t / 2\right]} \\
& \leq 1_{\left[\left(P_{n}^{\prime}-P_{n}\right) f_{n}>t / 2\right]}
\end{aligned}
$$

Taking expectations with respect to the second sample gives

$$
1_{\left[\left(P-P_{n}\right) f_{n}>t\right]} \mathbb{P}^{\prime}\left[\left(P-P_{n}^{\prime}\right) f_{n}<t / 2\right] \leq \mathbb{P}^{\prime}\left[\left(P_{n}^{\prime}-P_{n}\right) f_{n}>t / 2\right]
$$

O. Bousquet - Statistical Learning Theory - Lecture 3 


\section{Symmetrization (proof - 2)}

- By Chebyshev inequality,

$$
\mathbb{P}^{\prime}\left[\left(P-P_{n}^{\prime}\right) f_{n} \geq t / 2\right] \leq \frac{4 \operatorname{Var}\left[f_{n}\right]}{n t^{2}} \leq \frac{1}{n t^{2}}
$$

- Hence

$$
1_{\left[\left(P-P_{n}\right) f_{n}>t\right]}\left(1-\frac{1}{n t^{2}}\right) \leq \mathbb{P}^{\prime}\left[\left(P_{n}^{\prime}-P_{n}\right) f_{n}>t / 2\right]
$$

Take expectation with respect to first sample.

O. Bousquet - Statistical Learning Theory - Lecture 3 


\section{Proof of VC bound (1)}

- Symmetrization allows to replace expectation by average on ghost sample

- Function class projected on the double sample

$$
\mathcal{F}_{Z_{1}, \ldots, Z_{n}, Z_{1}^{\prime}, \ldots, Z_{n}^{\prime}}
$$

- Union bound on $\mathcal{F}_{Z_{1}, \ldots, Z_{n}, Z_{1}^{\prime}, \ldots, Z_{n}^{\prime}}$

- Variant of Hoeffding's inequality

$$
\mathbb{P}\left[P_{n} f-P_{n}^{\prime} f>t\right] \leq 2 e^{-n t^{2} / 2}
$$

O. Bousquet - Statistical Learning Theory - Lecture 3 


\section{Proof of VC bound (2)}

$$
\begin{aligned}
& \mathbb{P}\left[\sup _{f \in \mathcal{F}}\left(P-P_{n}\right) f \geq t\right] \\
& \leq 2 \mathbb{P}\left[\sup _{f \in \mathcal{F}}\left(P_{n}^{\prime}-P_{n}\right) f \geq t / 2\right] \\
& =2 \mathbb{P}\left[\sup _{f \in \mathcal{F}} Z_{Z_{1}, \ldots, Z_{n}, Z_{1}^{\prime}, \ldots, Z_{n}^{\prime}}\left(P_{n}^{\prime}-P_{n}\right) f \geq t / 2\right] \\
& \leq 2 S_{F}(2 n) \mathbb{P}\left[\left(P_{n}^{\prime}-P_{n}\right) f \geq t / 2\right] \\
& \leq 4 S_{F}(2 n) e^{-n t^{2} / 8}
\end{aligned}
$$

O. Bousquet - Statistical Learning Theory - Lecture 3 


\section{VC Entropy (1)}

- VC dimension is distribution independent

$\Rightarrow$ The same bound holds for any distribution

$\Rightarrow \mathrm{It}$ is loose for most distributions

- A similar proof can give a distribution-dependent result

O. Bousquet - Statistical Learning Theory - Lecture 3 


\section{VC Entropy (2)}

- Denote the size of the projection $N\left(\mathcal{F}, z_{1} \ldots, z_{n}\right):=\# \mathcal{F}_{z_{1}, \ldots, z_{n}}$

- The VC entropy is defined as

$$
H_{\mathcal{F}}(n)=\log \mathbb{E}\left[N\left(\mathcal{F}, Z_{1}, \ldots, Z_{n}\right)\right],
$$

- VC entropy bound: with probability at least $1-\delta$

$$
\forall g \in \mathcal{G}, R(g) \leq R_{n}(g)+\sqrt{\frac{H_{\mathcal{G}}(2 n)+\log \frac{2}{\delta}}{8 n}}
$$

O. Bousquet - Statistical Learning Theory - Lecture 3 


\section{VC Entropy (proof)}

Introduce $\sigma_{i} \in\{-1,1\}$ (probability $1 / 2$ ), Rademacher variables

$$
\begin{aligned}
2 \mathbb{P} & {\left[\sup _{f \in \mathcal{F}_{Z, Z^{\prime}}}\left(P_{n}^{\prime}-P_{n}\right) f \geq t / 2\right] } \\
& \leq 2 \mathbb{E}\left[\mathbb{P}_{\sigma}\left[\sup _{f \in \mathcal{F}_{Z, Z^{\prime}}} \frac{1}{n} \sum_{i=1}^{n} \sigma_{i}\left(f\left(Z_{i}^{\prime}\right)-f\left(Z_{i}\right)\right) \geq t / 2\right]\right] \\
& \leq 2 \mathbb{E}\left[N\left(\mathcal{F}, Z, Z^{\prime}\right)\right] \mathbb{P}\left[\frac{1}{n} \sum_{i=1}^{n} \sigma_{i} \geq t / 2\right] \\
& \leq 2 \mathbb{E}\left[N\left(\mathcal{F}, Z, Z^{\prime}\right)\right] e^{-n t^{2} / 8}
\end{aligned}
$$

O. Bousquet - Statistical Learning Theory - Lecture 3 


\section{From Bounds to Algorithms}

- For any distribution, $H_{\mathcal{G}}(n) / n \rightarrow 0$ ensures consistency of empirical risk minimizer (i.e. convergence to best in the class)

- Does it means we can learn anything ?

- No because of the approximation of the class

- Need to trade-off approximation and estimation error (assessed by the bound)

$\Rightarrow$ Use the bound to control the trade-off

O. Bousquet - Statistical Learning Theory - Lecture 3 


\section{Structural risk minimization}

- Structural risk minimization (SRM) (Vapnik, 1979): minimize the right hand side of

$$
R(g) \leq R_{n}(g)+B(h, n) .
$$

- To this end, introduce a structure on $\mathcal{G}$.

- Learning machine $\equiv$ a set of functions and an induction principle

O. Bousquet - Statistical Learning Theory - Lecture 3 


\section{SRM: The Picture}

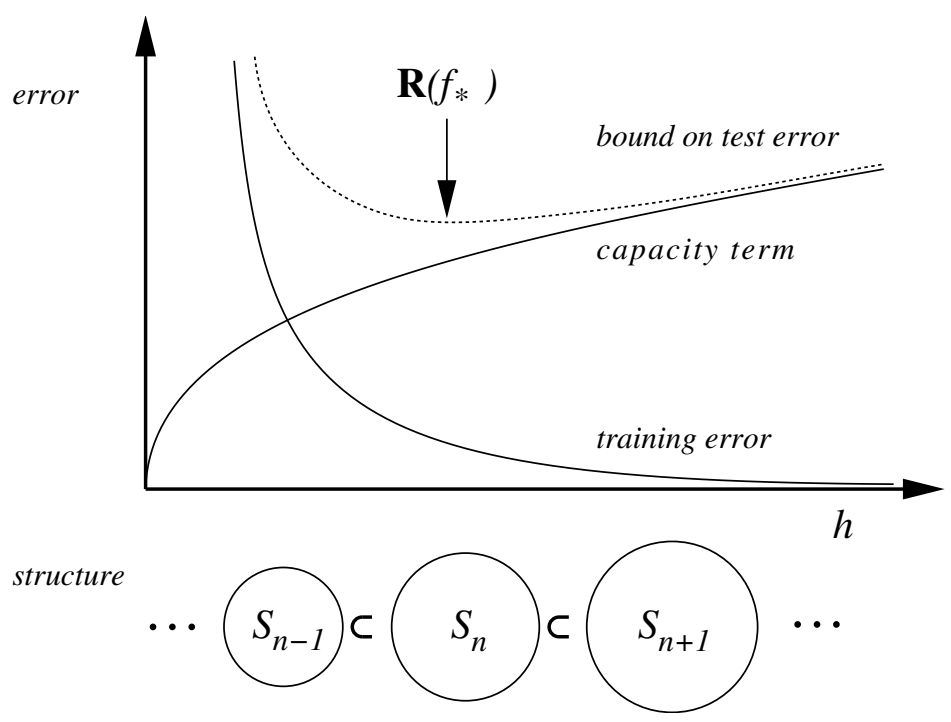

O. Bousquet - Statistical Learning Theory - Lecture 3 


\section{Lecture 4}

\section{Capacity Measures}

- Covering numbers

- Rademacher averages

- Relationships

O. Bousquet - Statistical Learning Theory - Lecture 4 


\section{Covering numbers}

- Define a (random) distance $d$ between functions, e.g.

$$
d\left(f, f^{\prime}\right)=\frac{1}{n} \#\left\{f\left(Z_{i}\right) \neq f^{\prime}\left(Z_{i}\right): i=1, \ldots, n\right\}
$$

Normalized Hamming distance of the 'projections' on the sample

- A set $f_{1}, \ldots, f_{N}$ covers $\mathcal{F}$ at radius $\varepsilon$ if

$$
\mathcal{F} \subset \cup_{i=1}^{N} B\left(f_{i}, \varepsilon\right)
$$

- Covering number $N(\mathcal{F}, \varepsilon, n)$ is the minimum size of a cover of radius $\varepsilon$

Note that $N(\mathcal{F}, \varepsilon, n)=N(\mathcal{G}, \varepsilon, n)$. 


\section{Bound with covering numbers}

- When the covering numbers are finite, one can approximate the class $\mathcal{G}$ by a finite set of functions

- Result

$$
\mathbb{P}\left[\exists g \in \mathcal{G}: R(g)-R_{n}(g) \geq t\right] \leq 8 \mathbb{E}[N(\mathcal{G}, t, n)] e^{-n t^{2} / 128}
$$

O. Bousquet - Statistical Learning Theory - Lecture 4 


\section{Covering numbers and VC dimension}

- Notice that for all $t, N(\mathcal{G}, t, n) \leq \# \mathcal{G}_{Z}=N(\mathcal{G}, Z)$

- Hence $N(\mathcal{G}, t, n) \leq h \log \frac{e n}{h}$

- Haussler

$$
N(\mathcal{G}, t, n) \leq C h(4 e)^{h} \frac{1}{t^{h}}
$$

- Independent of $n$

O. Bousquet - Statistical Learning Theory - Lecture 4 


\section{Refinement}

- VC entropy corresponds to log covering numbers at minimal scale

- Covering number bound is a generalization where the scale is adapted to the error

- Is this the right scale?

- It turns out that results can be improved by considering all scales $(\rightarrow$ chaining)

O. Bousquet - Statistical Learning Theory - Lecture 4 


\section{Rademacher averages}

- Rademacher variables: $\sigma_{1}, \ldots, \sigma_{n}$ independent random variables with

$$
\mathbb{P}\left[\sigma_{i}=1\right]=\mathbb{P}\left[\sigma_{i}=-1\right]=\frac{1}{2}
$$

- Notation (randomized empirical measure) $R_{n} f=\frac{1}{n} \sum_{i=1}^{n} \sigma_{i} f\left(Z_{i}\right)$

- Rademacher average: $\mathcal{R}(\mathcal{F})=\mathbb{E}\left[\sup _{f \in \mathcal{F}} R_{n} f\right]$

- Conditional Rademacher average $\mathcal{R}_{n}(\mathcal{F})=\mathbb{E}_{\sigma}\left[\sup _{f \in \mathcal{F}} R_{n} f\right]$ 


\section{Result}

- Distribution dependent

$$
\forall f \in \mathcal{F}, P f \leq P_{n} f+2 \mathcal{R}(\mathcal{F})+\sqrt{\frac{\log \frac{1}{\delta}}{2 n}},
$$

- Data dependent

$$
\forall f \in \mathcal{F}, P f \leq P_{n} f+2 \mathcal{R}_{n}(\mathcal{F})+\sqrt{\frac{2 \log \frac{2}{\delta}}{n}},
$$

O. Bousquet - Statistical Learning Theory - Lecture 4 


\section{Concentration}

- Hoeffding's inequality is a concentration inequality

- When $n$ increases, the average is concentrated around the expectation

- Generalization to functions that depend on i.i.d. random variables exist 


\section{McDiarmid's Inequality}

Assume for all $i=1, \ldots, n$,

$$
\sup _{z_{1}, \ldots, z_{n}, z_{i}^{\prime}}\left|F\left(z_{1}, \ldots, z_{i}, \ldots, z_{n}\right)-F\left(z_{1}, \ldots, z_{i}^{\prime}, \ldots, z_{n}\right)\right| \leq c
$$

then for all $\varepsilon>0$,

$$
\mathbb{P}[|F-\mathbb{E}[F]|>\varepsilon] \leq 2 \exp \left(-\frac{2 \varepsilon^{2}}{n c^{2}}\right)
$$

O. Bousquet - Statistical Learning Theory - Lecture 4 


\section{Proof of Rademacher average bounds}

- Use concentration to relate $\sup _{f \in \mathcal{F}} P f-P_{n} f$ to its expectation

- Use symmetrization to relate expectation to Rademacher average

- Use concentration again to relate Rademacher average to conditional one

O. Bousquet - Statistical Learning Theory - Lecture 4 


\section{Application (1)}

$$
\sup _{f \in \mathcal{F}} A(f)+B(f) \leq \sup _{f \in \mathcal{F}} A(f)+\sup _{f \in \mathcal{F}} B(f)
$$

Hence

$$
\left|\sup _{f \in \mathcal{F}} C(f)-\sup _{f \in \mathcal{F}} A(f)\right| \leq \sup _{f \in \mathcal{F}}(C(f)-A(f))
$$

this gives

$$
\left|\sup _{f \in \mathcal{F}}\left(P f-P_{n} f\right)-\sup _{f \in \mathcal{F}}\left(P f-P_{n}^{\prime} f\right)\right| \leq \sup _{f \in \mathcal{F}}\left(P_{n}^{\prime} f-P_{n} f\right)
$$

O. Bousquet - Statistical Learning Theory - Lecture 4 


\section{Application (2)}

$f \in\{0,1\}$ hence,

$$
P_{n}^{\prime} f-P_{n} f=\frac{1}{n}\left(f\left(Z_{i}^{\prime}\right)-f\left(Z_{i}\right)\right) \leq \frac{1}{n}
$$

thus

$$
\left|\sup _{f \in \mathcal{F}}\left(P f-P_{n} f\right)-\sup _{f \in \mathcal{F}}\left(P f-P_{n}^{\prime} f\right)\right| \leq \frac{1}{n}
$$

McDiarmid's inequality can be applied with $c=1 / n$

O. Bousquet - Statistical Learning Theory - Lecture 4 


\section{Symmetrization (1)}

- Upper bound

$$
\mathbb{E}\left[\sup _{f \in \mathcal{F}} P f-P_{n} f\right] \leq 2 \mathbb{E}\left[\sup _{f \in \mathcal{F}} R_{n} f\right]
$$

- Lower bound

$$
\mathbb{E}\left[\sup _{f \in \mathcal{F}}\left|P f-P_{n} f\right|\right] \geq \frac{1}{2} \mathbb{E}\left[\sup _{f \in \mathcal{F}} \mathcal{R}_{n} f\right]-\frac{1}{2 \sqrt{n}}
$$

O. Bousquet - Statistical Learning Theory - Lecture 4 


\section{Symmetrization (2)}

$$
\begin{aligned}
\mathbb{E}\left[\sup _{f \in \mathcal{F}} P f-P_{n} f\right] \\
\quad=\mathbb{E}\left[\sup _{f \in \mathcal{F}} \mathbb{E}\left[P_{n}^{\prime} f\right]-P_{n} f\right] \\
\leq \mathbb{E}_{Z, Z^{\prime}}\left[\sup _{f \in \mathcal{F}} P_{n}^{\prime} f-P_{n} f\right] \\
\quad=\mathbb{E}_{\sigma, Z, Z^{\prime}}\left[\sup _{f \in \mathcal{F}} \frac{1}{n} \sum_{i=1}^{n} \sigma_{i}\left(f\left(Z_{i}^{\prime}\right)-f\left(Z_{i}\right)\right)\right] \leq 2 \mathbb{E}\left[\sup _{f \in \mathcal{F}} R_{n} f\right]
\end{aligned}
$$




\section{Loss class and initial class}

$$
\begin{aligned}
\mathcal{R}(\mathcal{F}) & =\mathbb{E}\left[\sup _{g \in \mathcal{G}} \frac{1}{n} \sum_{i=1}^{n} \sigma_{i} 1_{\left[g\left(X_{i}\right) \neq Y_{i}\right]}\right] \\
& =\mathbb{E}\left[\sup _{g \in \mathcal{G}} \frac{1}{n} \sum_{i=1}^{n} \sigma_{i} \frac{1}{2}\left(1-Y_{i} g\left(X_{i}\right)\right)\right] \\
& =\frac{1}{2} \mathbb{E}\left[\sup _{g \in \mathcal{G}} \frac{1}{n} \sum_{i=1}^{n} \sigma_{i} Y_{i} g\left(X_{i}\right)\right]=\frac{1}{2} \mathcal{R}(\mathcal{G})
\end{aligned}
$$

O. Bousquet - Statistical Learning Theory - Lecture 4 


\section{Computing Rademacher averages (1)}

$$
\begin{aligned}
\frac{1}{2} \mathbb{E} & {\left[\sup _{g \in \mathcal{G}} \frac{1}{n} \sum_{i=1}^{n} \sigma_{i} g\left(X_{i}\right)\right] } \\
& =\frac{1}{2}+\mathbb{E}\left[\sup _{g \in \mathcal{G}} \frac{1}{n} \sum_{i=1}^{n}-\frac{1-\sigma_{i} g\left(X_{i}\right)}{2}\right] \\
& =\frac{1}{2}-\mathbb{E}\left[\inf _{g \in \mathcal{G}} \frac{1}{n} \sum_{i=1}^{n} \frac{1-\sigma_{i} g\left(X_{i}\right)}{2}\right] \\
& =\frac{1}{2}-\mathbb{E}\left[\inf _{g \in \mathcal{G}} R_{n}(g, \sigma)\right]
\end{aligned}
$$

O. Bousquet - Statistical Learning Theory - Lecture 4 


\section{Computing Rademacher averages (2)}

- Not harder than computing empirical risk minimizer

- Pick $\sigma_{i}$ randomly and minimize error with respect to labels $\sigma_{i}$

- Intuition: measure how much the class can fit random noise

- Large class $\Rightarrow \mathcal{R}(\mathcal{G})=\frac{1}{2}$

O. Bousquet - Statistical Learning Theory - Lecture 4 


\section{Concentration again}

- Let

$$
F=\mathbb{E}_{\sigma}\left[\sup _{f \in \mathcal{F}} R_{n} f\right]
$$

Expectation with respect to $\sigma_{i}$ only, with $\left(X_{i}, Y_{i}\right)$ fixed.

- $F$ satisfies McDiarmid's assumptions with $c=\frac{1}{n}$

$\Rightarrow \mathbb{E}[F]=\mathcal{R}(\mathcal{F})$ can be estimated by $F=\mathcal{R}_{n}(\mathcal{F})$

O. Bousquet - Statistical Learning Theory - Lecture 4 


\section{Relationship with VC dimension}

- For a finite set $\mathcal{F}=\left\{f_{1}, \ldots, f_{N}\right\}$

$$
\mathcal{R}(\mathcal{F}) \leq 2 \sqrt{\log N / n}
$$

- Consequence for VC class $\mathcal{F}$ with dimension $h$

$$
\mathcal{R}(\mathcal{F}) \leq 2 \sqrt{\frac{h \log \frac{e n}{h}}{n}} .
$$

$\Rightarrow$ Recovers VC bound with a concentration proof

O. Bousquet - Statistical Learning Theory - Lecture 4 


\section{Chaining}

- Using covering numbers at all scales, the geometry of the class is better captured

- Dudley

$$
\mathcal{R}_{n}(\mathcal{F}) \leq \frac{C}{\sqrt{n}} \int_{0}^{\infty} \sqrt{\log N(\mathcal{F}, t, n)} d t
$$

- Consequence

$$
\mathcal{R}(\mathcal{F}) \leq C \sqrt{\frac{h}{n}}
$$

- Removes the unnecessary $\log n$ factor!

O. Bousquet - Statistical Learning Theory - Lecture 4 


\section{Lecture 5}

\section{Advanced Topics}

- Relative error bounds

- Noise conditions

- Localized Rademacher averages

- PAC-Bayesian bounds

O. Bousquet - Statistical Learning Theory - Lecture 5 


\section{Binomial tails}

- $P_{n} f \sim B(p, n)$ binomial distribution $p=P f$

- $\mathbb{P}\left[P f-P_{n} f \geq t\right]=\sum_{k=0}^{\lfloor n(p-t)\rfloor}\left(\begin{array}{l}n \\ k\end{array}\right) p^{k}(1-p)^{n-k}$

- Can be upper bounded

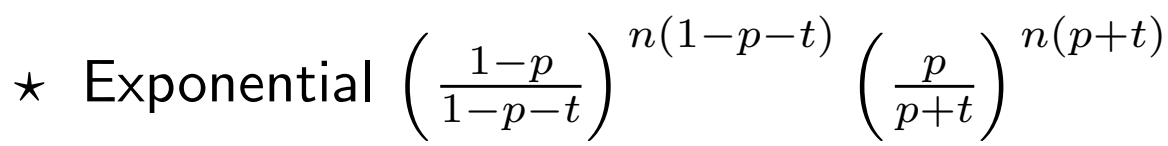

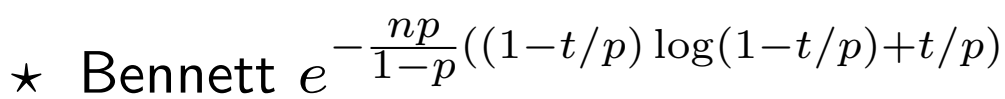

$\star$ Bernstein $e^{-\frac{n t^{2}}{2 p(1-p)+2 t / 3}}$

$\star$ Hoeffding $e^{-2 n t^{2}}$

O. Bousquet - Statistical Learning Theory - Lecture 5 


\section{Tail behavior}

- For small deviations, Gaussian behavior $\approx \exp \left(-n t^{2} / 2 p(1-p)\right)$ $\Rightarrow$ Gaussian with variance $p(1-p)$

- For large deviations, Poisson behavior $\approx \exp (-3 n t / 2)$

$\Rightarrow$ Tails heavier than Gaussian

- Can upper bound with a Gaussian with large (maximum) variance $\exp \left(-2 n t^{2}\right)$ 


\section{Illustration (1)}

Maximum variance $(p=0.5)$

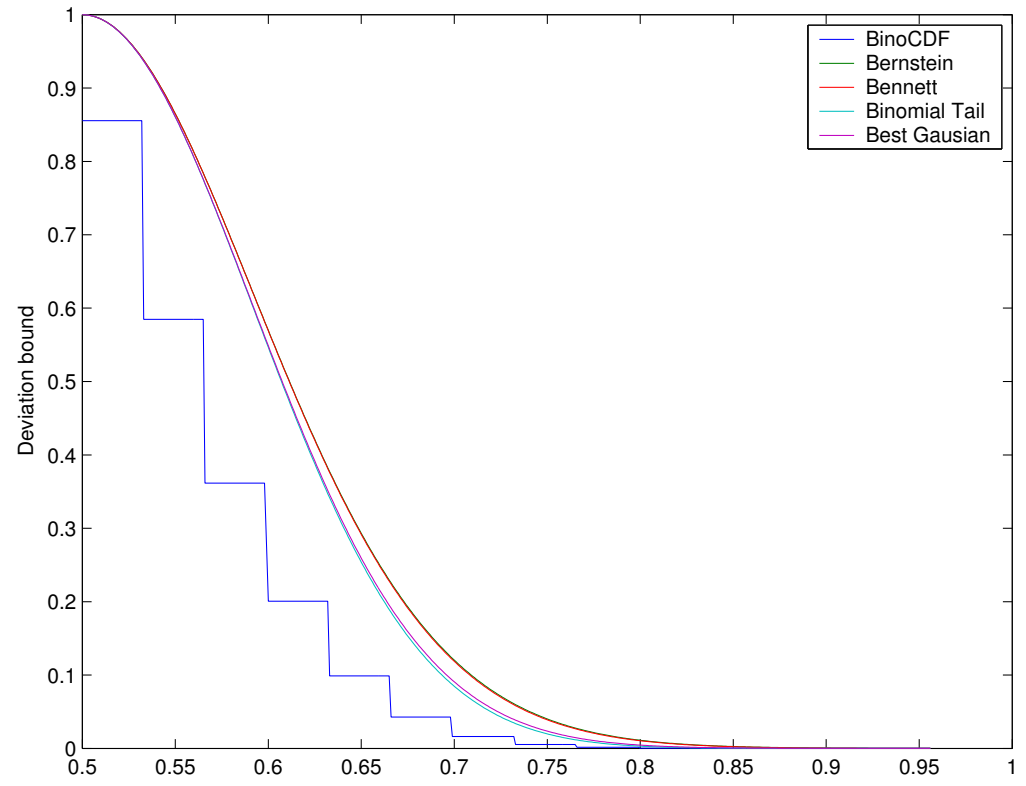

O. Bousquet - Statistical Learning Theory - Lecture 5 


\section{Illustration (2)}

Small variance $(p=0.1)$
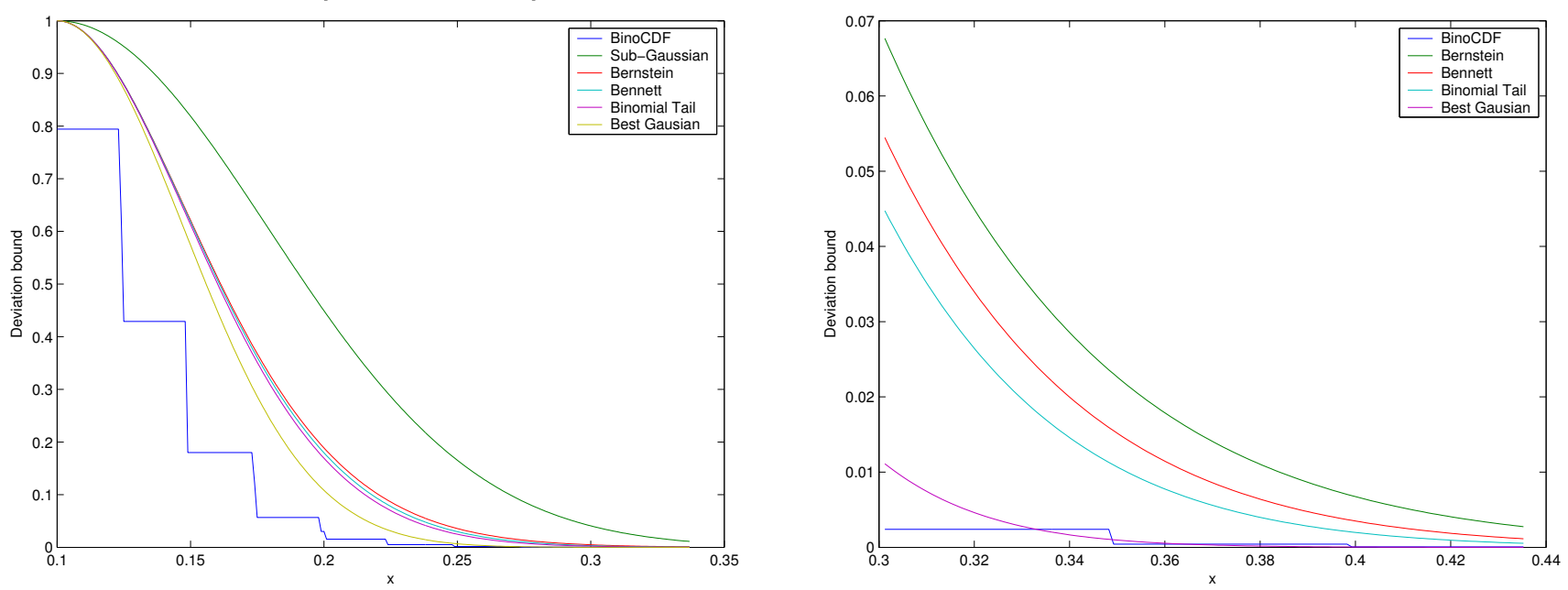

O. Bousquet - Statistical Learning Theory - Lecture 5 


\section{Taking the variance into account (1)}

- Each function $f \in \mathcal{F}$ has a different variance $P f(1-P f) \leq P f$.

- For each $f \in \mathcal{F}$, by Bernstein's inequality

$$
P f \leq P_{n} f+\sqrt{\frac{2 P f \log \frac{1}{\delta}}{n}}+\frac{2 \log \frac{1}{\delta}}{3 n}
$$

- The Gaussian part dominates (for $P f$ not too small, or $n$ large enough), it depends on $P f$ 


\section{Taking the variance into account (2)}

- Central Limit Theorem

$$
\sqrt{n} \frac{P f-P_{n} f}{\sqrt{P f(1-P f)}} \rightarrow N(0,1)
$$

$\Rightarrow$ Idea is to consider the ratio

$$
\frac{P f-P_{n} f}{\sqrt{P f}}
$$

O. Bousquet - Statistical Learning Theory - Lecture 5 


\section{Normalization}

- Here $(f \in\{0,1\}), \operatorname{Var}[f] \leq P f^{2}=P f$

- Large variance $\Rightarrow$ large risk.

- After normalization, fluctuations are more "uniform"

$$
\sup _{f \in \mathcal{F}} \frac{P f-P_{n} f}{\sqrt{P f}}
$$

not necessarily attained at functions with large variance.

- Focus of learning: functions with small error $\operatorname{Pf}$ (hence small variance).

$\Rightarrow$ The normalized supremum takes this into account. 


\section{Relative deviations}

Vapnik-Chervonenkis 1974

For $\delta>0$ with probability at least $1-\delta$,

$$
\forall f \in \mathcal{F}, \frac{P f-P_{n} f}{\sqrt{P f}} \leq 2 \sqrt{\frac{\log S_{\mathcal{F}}(2 n)+\log \frac{4}{\delta}}{n}}
$$

and

$$
\forall f \in \mathcal{F}, \frac{P_{n} f-P f}{\sqrt{P_{n} f}} \leq 2 \sqrt{\frac{\log S_{\mathcal{F}}(2 n)+\log \frac{4}{\delta}}{n}}
$$

O. Bousquet - Statistical Learning Theory - Lecture 5 


\section{Proof sketch}

1. Symmetrization

$$
\mathbb{P}\left[\sup _{f \in \mathcal{F}} \frac{P f-P_{n} f}{\sqrt{P f}} \geq t\right] \leq 2 \mathbb{P}\left[\sup _{f \in \mathcal{F}} \frac{P_{n}^{\prime} f-P_{n} f}{\sqrt{\left(P_{n} f+P_{n}^{\prime} f\right) / 2}} \geq t\right]
$$

2. Randomization

$$
\cdots=2 \mathbb{E}\left[\mathbb{P}_{\sigma}\left[\sup _{f \in \mathcal{F}} \frac{\frac{1}{n} \sum_{i=1}^{n} \sigma_{i}\left(f\left(Z_{i}^{\prime}\right)-f\left(Z_{i}\right)\right)}{\sqrt{\left(P_{n} f+P_{n}^{\prime} f\right) / 2}} \geq t\right]\right]
$$

3. Tail bound

O. Bousquet - Statistical Learning Theory - Lecture 5 


\section{Consequences}

From the fact

$$
A \leq B+C \sqrt{A} \Rightarrow A \leq B+C^{2}+\sqrt{B} C
$$

we get

$$
\begin{aligned}
\forall f \in \mathcal{F}, P f \leq & P_{n} f+2 \sqrt{P_{n} f \frac{\log S_{\mathcal{F}}(2 n)+\log \frac{4}{\delta}}{n}} \\
& +4 \frac{\log S_{\mathcal{F}}(2 n)+\log \frac{4}{\delta}}{n}
\end{aligned}
$$

O. Bousquet - Statistical Learning Theory - Lecture 5 


\section{Zero noise}

Ideal situation

- $g_{n}$ empirical risk minimizer

- $t \in \mathcal{G}$

- $R^{*}=0$ (no noise, $n(X)=0$ a.s.)

In that case

- $R_{n}\left(g_{n}\right)=0$

$\Rightarrow R\left(g_{n}\right)=O\left(\frac{d \log n}{n}\right)$.

O. Bousquet - Statistical Learning Theory - Lecture 5 


\section{Interpolating between rates?}

- Rates are not correctly estimated by this inequality

- Consequence of relative error bounds

$$
\begin{aligned}
P f_{n} \leq & P f^{*}+2 \sqrt{P f^{*} \frac{\log S_{\mathcal{F}}(2 n)+\log \frac{4}{\delta}}{n}} \\
& +4 \frac{\log S_{\mathcal{F}}(2 n)+\log \frac{4}{\delta}}{n}
\end{aligned}
$$

- The quantity which is small is not $P f^{*}$ but $P f_{n}-P f^{*}$

- But relative error bounds do not apply to differences 


\section{Definitions}

- $P=P_{X} \times P(Y \mid X)$

- regression function $\eta(x)=\mathbb{E}[Y \mid X=x]$

- target function $t(x)=\operatorname{sgn} \eta(x)$

- noise level $n(X)=(1-|\eta(x)|) / 2$

- Bayes risk $R^{*}=\mathbb{E}[n(X)]$

- $R(g)=\mathbb{E}[(1-\eta(X)) / 2]+\mathbb{E}\left[\eta(X) 1_{[g \leq 0]}\right]$

- $R(g)-R^{*}=\mathbb{E}\left[|\eta(X)| 1_{[g \eta \leq 0]}\right]$

O. Bousquet - Statistical Learning Theory - Lecture 5 


\section{Intermediate noise}

Instead of assuming that $|\eta(x)|=1$ (i.e. $n(x)=0$ ), the deterministic case, one can assume that $n$ is well-behaved. Two kinds of assumptions

- $n$ not too close to $1 / 2$

- $n$ not often too close to $1 / 2$

O. Bousquet - Statistical Learning Theory - Lecture 5 


\section{Massart Condition}

- For some $c>0$, assume

$$
|\eta(X)|>\frac{1}{c} \text { almost surely }
$$

- There is no region where the decision is completely random

- Noise bounded away from $1 / 2$

O. Bousquet - Statistical Learning Theory - Lecture 5 


\section{Tsybakov Condition}

Let $\alpha \in[0,1]$, equivalent conditions

$$
\begin{array}{ll}
\text { (1) } \quad \exists c>0, \forall g \in\{-1,1\}^{\mathcal{X}}, \\
& \mathbb{P}[g(X) \eta(X) \leq 0] \leq c\left(R(g)-R^{*}\right)^{\alpha} \\
\text { (2) } \quad \exists c>0, \forall A \subset \mathcal{X}, \int_{A} d P(x) \leq c\left(\int_{A}|\eta(x)| d P(x)\right)^{\alpha} \\
\text { (3) } \quad \exists B>0, \forall t \geq 0, \mathbb{P}[|\eta(X)| \leq t] \leq B t^{\frac{\alpha}{1-\alpha}}
\end{array}
$$




\section{Equivalence}

- (1) $\Leftrightarrow(2)$ Recall $R(g)-R^{*}=\mathbb{E}\left[|\eta(X)| 1_{[g \eta \leq 0]}\right]$. For each function $g$, there exists a set $A$ such that $1_{[A]}=1_{[g \eta \leq 0]}$

- (2) $\Rightarrow(3)$ Let $A=\{x:|\eta(x)| \leq t\}$

$$
\begin{aligned}
\mathbb{P}[|\eta| \leq t]=\int_{A} d P(x) & \leq c\left(\int_{A}|\eta(x)| d P(x)\right)^{\alpha} \\
& \leq c t^{\alpha}\left(\int_{A} d P(x)\right)^{\alpha} \\
& \Rightarrow \mathbb{P}[|\eta| \leq t] \leq c^{\frac{1}{1-\alpha}} t^{\frac{\alpha}{1-\alpha}}
\end{aligned}
$$

O. Bousquet - Statistical Learning Theory - Lecture 5 
- $(3) \Rightarrow(1)$

$$
\begin{aligned}
& R(g)-R^{*}=\mathbb{E}\left[|\eta(X)| 1_{[g \eta \leq 0]}\right] \\
& \quad \geq t \mathbb{E}\left[1_{[g \eta \leq 0]} 1_{[|\eta|>t]}\right] \\
& \quad=t \mathbb{P}[|\eta|>t]-t \mathbb{E}\left[1_{[g \eta>0]} 1_{[|\eta|>t]}\right] \\
& \quad \geq t\left(1-B t^{\frac{\alpha}{1-\alpha}}\right)-t \mathbb{P}[g \eta>0]=t\left(\mathbb{P}[g \eta \leq 0]-B t^{\frac{\alpha}{1-\alpha}}\right)
\end{aligned}
$$

Take $t=\left(\frac{(1-\alpha) \mathbb{P}[g \eta \leq 0]}{B}\right)^{(1-\alpha) / \alpha}$

$$
\Rightarrow \mathbb{P}[g \eta \leq 0] \leq \frac{B^{1-\alpha}}{(1-\alpha)^{(1-\alpha) \alpha^{\alpha}}}\left(R(g)-R^{*}\right)^{\alpha}
$$

O. Bousquet - Statistical Learning Theory - Lecture 5 


\section{Remarks}

- $\alpha$ is in $[0,1]$ because

$$
R(g)-R^{*}=\mathbb{E}\left[|\eta(X)| 1_{[g \eta \leq 0]}\right] \leq \mathbb{E}\left[1_{[g \eta \leq 0]}\right]
$$

- $\alpha=0$ no condition

- $\alpha=1$ gives Massart's condition

O. Bousquet - Statistical Learning Theory - Lecture 5 


\section{Consequences}

- Under Massart's condition

$$
\mathbb{E}\left[\left(1_{[g(X) \neq Y]}-1_{[t(X) \neq Y]}\right)^{2}\right] \leq c\left(R(g)-R^{*}\right)
$$

- Under Tsybakov's condition

$$
\mathbb{E}\left[\left(1_{[g(X) \neq Y]}-1_{[t(X) \neq Y]}\right)^{2}\right] \leq c\left(R(g)-R^{*}\right)^{\alpha}
$$

O. Bousquet - Statistical Learning Theory - Lecture 5 


\section{Relative loss class}

- $\mathcal{F}$ is the loss class associated to $\mathcal{G}$

- The relative loss class is defined as

$$
\tilde{\mathcal{F}}=\left\{f-f^{*}: f \in \mathcal{F}\right\}
$$

- It satisfies

$$
P f^{2} \leq c(P f)^{\alpha}
$$

O. Bousquet - Statistical Learning Theory - Lecture 5 


\section{Finite case}

- Union bound on $\tilde{\mathcal{F}}$ with Bernstein's inequality would give

$$
P f_{n}-P f^{*} \leq P_{n} f_{n}-P_{n} f^{*}+\sqrt{\frac{8 c\left(P f_{n}-P f^{*}\right)^{\alpha} \log \frac{N}{\delta}}{n}}+\frac{4 \log \frac{N}{\delta}}{3 n}
$$

- Consequence when $f^{*} \in \mathcal{F}$ (but $R^{*}>0$ )

$$
P f_{n}-P f^{*} \leq C\left(\frac{\log \frac{N}{\delta}}{n}\right)^{\frac{1}{2-\alpha}}
$$

always better than $n^{-1 / 2}$ for $\alpha>0$

O. Bousquet - Statistical Learning Theory - Lecture 5 


\section{Local Rademacher average}

- Definition

$$
\mathcal{R}(\mathcal{F}, r)=\mathbb{E}\left[\sup _{f \in \mathcal{F}: P f^{2} \leq r} R_{n} f\right]
$$

- Allows to generalize the previous result

- Computes the capacity of a small ball in $\mathcal{F}$ (functions with small variance)

- Under noise conditions, small variance implies small error

O. Bousquet - Statistical Learning Theory - Lecture 5 


\section{Sub-root functions}

\section{Definition}

A function $\psi: \mathbb{R} \rightarrow \mathbb{R}$ is sub-root if

- $\psi$ is non-decreasing

- $\psi$ is non negative

- $\psi(r) / \sqrt{r}$ is non-increasing

O. Bousquet - Statistical Learning Theory - Lecture 5 


\section{Sub-root functions}

\section{Properties}

A sub-root function

- is continuous

- has a unique fixed point $\psi\left(r^{*}\right)=r^{*}$

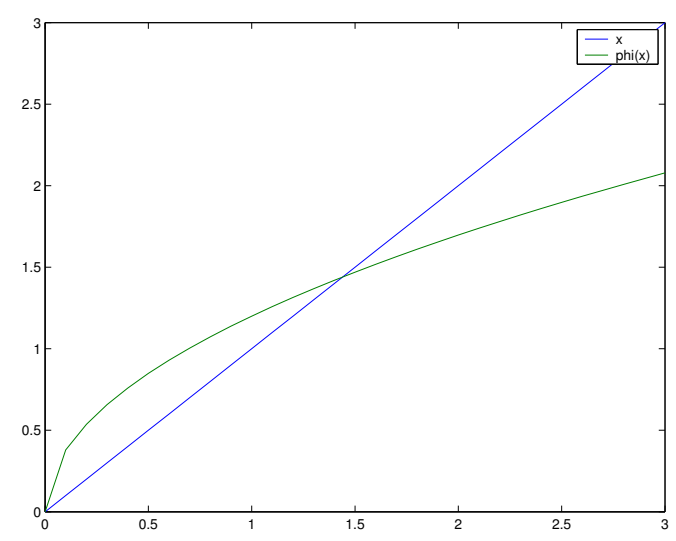

O. Bousquet - Statistical Learning Theory - Lecture 5 


\section{Star hull}

- Definition

$$
\star \mathcal{F}=\{\alpha f: f \in \mathcal{F}, \alpha \in[0,1]\}
$$

- Properties

$$
\mathcal{R}_{n}(\star \mathcal{F}, r) \text { is sub-root }
$$

- Entropy of $\star \mathcal{F}$ is not much bigger than entropy of $\mathcal{F}$

O. Bousquet - Statistical Learning Theory - Lecture 5 


\section{Result}

- $r^{*}$ fixed point of $\mathcal{R}(\star \mathcal{F}, r)$

- Bounded functions

$$
P f-P_{n} f \leq C\left(\sqrt{r^{*} \operatorname{Var}[f]}+\frac{\log \frac{1}{\delta}+\log \log n}{n}\right)
$$

- Consequence for variance related to expectation $\left(\operatorname{Var}[f] \leq c(P f)^{\beta}\right)$

$$
P f \leq C\left(P_{n} f+\left(r^{*}\right)^{\frac{1}{2-\beta}}+\frac{\log \frac{1}{\delta}+\log \log n}{n}\right)
$$

O. Bousquet - Statistical Learning Theory - Lecture 5 


\section{Consequences}

- For VC classes $\mathcal{R}(\mathcal{F}, r) \leq C \sqrt{\frac{\overline{r h}}{n}}$ hence $r^{*} \leq C \frac{h}{n}$

- Rate of convergence of $P_{n} f$ to $P f$ in $O(1 / \sqrt{n})$

- But rate of convergence of $P f_{n}$ to $P f^{*}$ is $O\left(1 / n^{1 /(2-\alpha)}\right)$

Only condition is $t \in \mathcal{G}$ but can be removed by SRM/Model selection 


\section{Proof sketch (1)}

- Talagrand's inequality

$$
\sup _{f \in \mathcal{F}} P f-P_{n} f \leq \mathbb{E}\left[\sup _{f \in \mathcal{F}} P f-P_{n} f\right]+c \sqrt{\sup _{f \in \mathcal{F}} \operatorname{Var}[f] / n}+c^{\prime} / n
$$

- Peeling of the class

$$
\mathcal{F}_{k}=\left\{f: \operatorname{Var}[f] \in\left[x^{k}, x^{k+1}\right)\right\}
$$

O. Bousquet - Statistical Learning Theory - Lecture 5 


\section{Proof sketch (2)}

- Application

$$
\sup _{f \in \mathcal{F}_{k}} P f-P_{n} f \leq \mathbb{E}\left[\sup _{f \in \mathcal{F}_{k}} P f-P_{n} f\right]+c \sqrt{x \operatorname{Var}[f] / n}+c^{\prime} / n
$$

- Symmetrization

$$
\forall f \in \mathcal{F}, P f-P_{n} f \leq 2 \mathcal{R}(\mathcal{F}, x \operatorname{Var}[f])+c \sqrt{x \operatorname{Var}[f] / n}+c^{\prime} / n
$$

O. Bousquet - Statistical Learning Theory - Lecture 5 


\section{Proof sketch (3)}

- We need to 'solve' this inequality. Things are simple if $\mathcal{R}$ behave like a square root, hence the sub-root property

$$
P f-P_{n} f \leq 2 \sqrt{r^{*} \operatorname{Var}[f]}+c \sqrt{x \operatorname{Var}[f] / n}+c^{\prime} / n
$$

- Variance-expectation

$$
\operatorname{Var}[f] \leq c(P f)^{\alpha}
$$

Solve in $P f$

O. Bousquet - Statistical Learning Theory - Lecture 5 


\section{Data-dependent version}

- As in the global case, one can use data-dependent local Rademcher averages

$$
\mathcal{R}_{n}(\mathcal{F}, r)=\mathbb{E}_{\sigma}\left[\sup _{f \in \mathcal{F}: P f^{2} \leq r} R_{n} f\right]
$$

- Using concentration one can also get

$$
P f \leq C\left(P_{n} f+\left(r_{n}^{*}\right)^{\frac{1}{2-\alpha}}+\frac{\log \frac{1}{\delta}+\log \log n}{n}\right)
$$

where $r_{n}^{*}$ is the fixed point of a sub-root upper bound of $\mathcal{R}_{n}(\mathcal{F}, r)$

O. Bousquet - Statistical Learning Theory - Lecture 5 


\section{Discussion}

- Improved rates under low noise conditions

- Interpolation in the rates

- Capacity measure seems 'local',

- but depends on all the functions,

- after appropriate rescaling: each $f \in \mathcal{F}$ is considered at scale $r / P f^{2}$

O. Bousquet - Statistical Learning Theory - Lecture 5 


\section{Randomized Classifiers}

Given $\mathcal{G}$ a class of functions

- Deterministic: picks a function $g_{n}$ and always use it to predict

- Randomized

* construct a distribution $\rho_{n}$ over $\mathcal{G}$

* for each instance to classify, pick $g \sim \rho_{n}$

- Error is averaged over $\rho_{n}$

$$
\begin{gathered}
R\left(\rho_{n}\right)=\rho_{n} P f \\
R_{n}\left(\rho_{n}\right)=\rho_{n} P_{n} f
\end{gathered}
$$

O. Bousquet - Statistical Learning Theory - Lecture 5 


\section{Union Bound (1)}

Let $\pi$ be a (fixed) distribution over $\mathcal{F}$.

- Recall the refined union bound

$$
\forall f \in \mathcal{F}, P f-P_{n} f \leq \sqrt{\frac{\log \frac{1}{\pi(f)}+\log \frac{1}{\delta}}{2 n}}
$$

- Take expectation with respect to $\rho_{n}$

$$
\rho_{n} P f-\rho_{n} P_{n} f \leq \rho_{n} \sqrt{\frac{\log \frac{1}{\pi(f)}+\log \frac{1}{\delta}}{2 n}}
$$

O. Bousquet - Statistical Learning Theory - Lecture 5 


\section{Union Bound (2)}

$$
\begin{aligned}
\rho_{n} P f-\rho_{n} P_{n} f & \leq \rho_{n} \sqrt{\left(-\log \pi(f)+\log \frac{1}{\delta}\right) /(2 n)} \\
& \leq \sqrt{\left(-\rho_{n} \log \pi(f)+\log \frac{1}{\delta}\right) /(2 n)} \\
& \leq \sqrt{\left(K\left(\rho_{n}, \pi\right)+H\left(\rho_{n}\right)+\log \frac{1}{\delta}\right) /(2 n)}
\end{aligned}
$$

- $K\left(\rho_{n}, \pi\right)=\int \rho_{n}(f) \log \frac{\rho_{n}(f)}{\pi(f)} d f$ Kullback-Leibler divergence

- $H\left(\rho_{n}\right)=\int \rho_{n}(f) \log \rho_{n}(f) d f$ Entropy 


\section{PAC-Bayesian Refinement}

- It is possible to improve the previous bound.

- With probability at least $1-\delta$,

$$
\rho_{n} P f-\rho_{n} P_{n} f \leq \sqrt{\frac{K\left(\rho_{n}, \pi\right)+\log 4 n+\log \frac{1}{\delta}}{2 n-1}}
$$

- Good if $\rho_{n}$ is spread (i.e. large entropy)

- Not interesting if $\rho_{n}=\delta_{f_{n}}$

O. Bousquet - Statistical Learning Theory - Lecture 5 


\section{Proof (1)}

- Variational formulation of entropy: for any $T$

$$
\rho T(f) \leq \log \pi e^{T(f)}+K(\rho, \pi)
$$

- Apply it to $\lambda\left(P f-P_{n} f\right)^{2}$

$$
\lambda \rho_{n}\left(P f-P_{n} f\right)^{2} \leq \log \pi e^{\lambda(P f-P n f)^{2}}+K\left(\rho_{n}, \pi\right)
$$

- Markov's inequality: with probability $1-\delta$,

$$
\lambda \rho_{n}\left(P f-P_{n} f\right)^{2} \leq \log \mathbb{E}\left[\pi e^{\lambda\left(P f-P_{n} f\right)^{2}}\right]+K\left(\rho_{n}, \pi\right)+\log \frac{1}{\delta}
$$

O. Bousquet - Statistical Learning Theory - Lecture 5 


\section{Proof (2)}

- Fubini

$$
\mathbb{E}\left[\pi e^{\lambda\left(P f-P_{n} f\right)^{2}}\right]=\pi \mathbb{E}\left[e^{\lambda\left(P f-P_{n} f\right)^{2}}\right]
$$

- Modified Chernoff bound

$$
\mathbb{E}\left[e^{(2 n-1)\left(P f-P_{n} f\right)^{2}}\right] \leq 4 n
$$

- Putting together $(\lambda=2 n-1)$

$$
(2 n-1) \rho_{n}\left(P f-P_{n} f\right)^{2} \leq K\left(\rho_{n}, \pi\right)+\log 4 n+\log \frac{1}{\delta}
$$

- Jensen $(2 n-1)\left(\rho_{n}\left(P f-P_{n} f\right)\right)^{2} \leq(2 n-1) \rho_{n}\left(P f-P_{n} f\right)^{2}$

O. Bousquet - Statistical Learning Theory - Lecture 5 


\section{Lecture 6}

\section{Loss Functions}

- Properties

- Consistency

- Examples

- Losses and noise

O. Bousquet - Statistical Learning Theory - Lecture 6 


\section{Motivation (1)}

- ERM: minimize $\sum_{i=1}^{n} 1_{\left[g\left(X_{i}\right) \neq Y_{i}\right]}$ in a set $\mathcal{G}$

$\Rightarrow$ Computationally hard

$\Rightarrow$ Smoothing

* Replace binary by real-valued functions

* Introduce smooth loss function

$$
\sum_{i=1}^{n} \ell\left(g\left(X_{i}\right), Y_{i}\right)
$$

O. Bousquet - Statistical Learning Theory - Lecture 6 


\section{Motivation (2)}

- Hyperplanes in infinite dimension have

$\star$ infinite VC-dimension

$\star$ but finite scale-sensitive dimension (to be defined later)

$\Rightarrow$ It is good to have a scale

$\Rightarrow$ This scale can be used to give a confidence (i.e. estimate the density)

- However, losses do not need to be related to densities

- Can get bounds in terms of margin error instead of empirical error (smoother $\rightarrow$ easier to optimize for model selection) 


\section{Margin}

- It is convenient to work with (symmetry of +1 and -1 )

$$
\ell(g(x), y)=\phi(y g(x))
$$

- $y g(x)$ is the margin of $g$ at $(x, y)$

- Loss

$$
L(g)=\mathbb{E}[\phi(Y g(X))], L_{n}(g)=\frac{1}{n} \sum_{i=1}^{n} \phi\left(Y_{i} g\left(X_{i}\right)\right)
$$

- Loss class $\mathcal{F}=\{f:(x, y) \mapsto \phi(y g(x)): g \in \mathcal{G}\}$

O. Bousquet - Statistical Learning Theory - Lecture 6 


\section{Minimizing the loss}

- Decomposition of $L(g)$

$$
\frac{1}{2} \mathbb{E}[\mathbb{E}[(1+\eta(X)) \phi(g(X))+(1-\eta(X)) \phi(-g(X)) \mid X]]
$$

- Minimization for each $x$

$$
H(\eta)=\inf _{\alpha \in \mathbb{R}}((1+\eta) \phi(\alpha) / 2+(1-\eta) \phi(-\alpha) / 2)
$$

- $L^{*}:=\inf _{g} L(g)=\mathbb{E}[H(\eta(X))]$ 


\section{Classification-calibrated}

- A minimal requirement is that the minimizer in $H(\eta)$ has the correct sign (that of the target $t$ or that of $\eta$ ).

- Definition

$\phi$ is classification-calibrated if, for any $\eta \neq 0$

$\inf _{\alpha: \alpha \eta \leq 0}(1+\eta) \phi(\alpha)+(1-\eta) \phi(-\alpha)>\inf _{\alpha \in \mathbb{R}}(1+\eta) \phi(\alpha)+(1-\eta) \phi(-\alpha)$

- This means the infimum is achieved for an $\alpha$ of the correct sign (and not for an $\alpha$ of the wrong sign, except possibly for $\eta=0$ ).

O. Bousquet - Statistical Learning Theory - Lecture 6 


\section{Consequences (1)}

Results due to (Jordan, Bartlett and McAuliffe 2003)

- $\phi$ is classification-calibrated iff for all sequences $g_{i}$ and every probability distribution $P$,

$$
L\left(g_{i}\right) \rightarrow L^{*} \Rightarrow R\left(g_{i}\right) \rightarrow R^{*}
$$

- When $\phi$ is convex (convenient for optimization) $\phi$ is classificationcalibrated iff it is differentiable at 0 and $\phi^{\prime}(0)<0$

O. Bousquet - Statistical Learning Theory - Lecture 6 


\section{Consequences (2)}

- Let $H^{-}(\eta)=\inf _{\alpha: \alpha \eta \leq 0}((1+\eta) \phi(\alpha) / 2+(1-\eta) \phi(-\alpha) / 2)$

- Let $\psi(\eta)$ be the largest convex function below $H^{-}(\eta)-H(\eta)$

- One has

$$
\psi\left(R(g)-R^{*}\right) \leq L(g)-L^{*}
$$

O. Bousquet - Statistical Learning Theory - Lecture 6 


\section{Examples (1)}

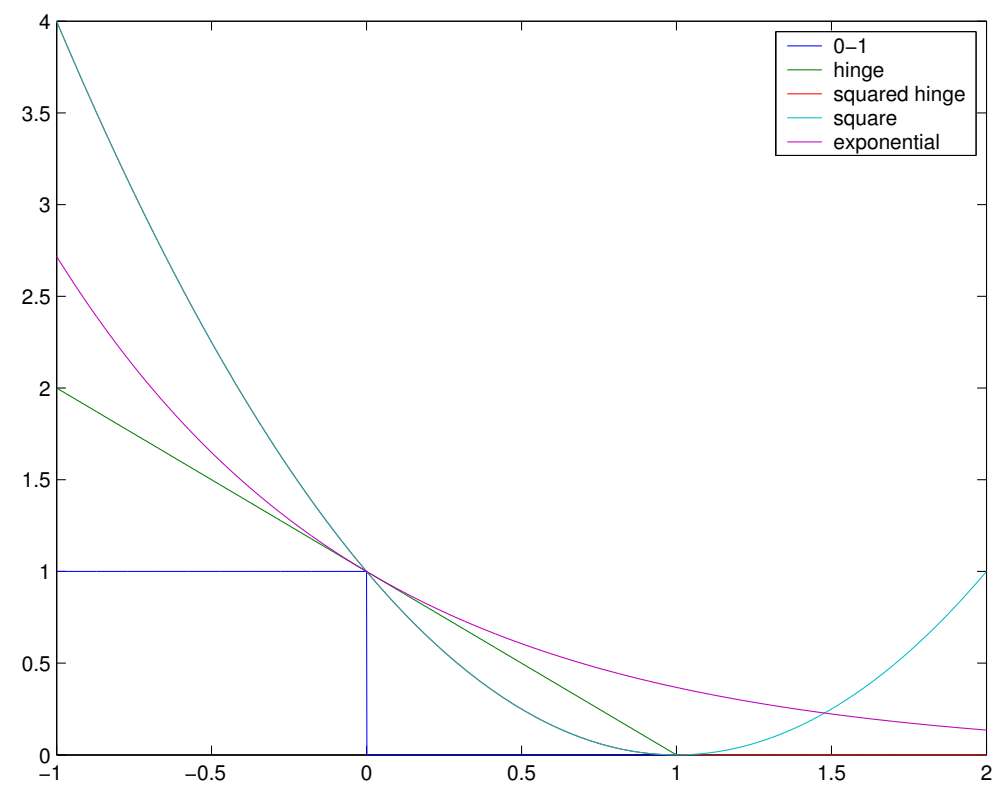

O. Bousquet - Statistical Learning Theory - Lecture 6 


\section{Examples (2)}

- Hinge loss

$$
\phi(x)=\max (0,1-x), \psi(x)=x
$$

- Squared hinge loss

$$
\phi(x)=\max (0,1-x)^{2}, \psi(x)=x^{2}
$$

- Square loss

$$
\phi(x)=(1-x)^{2}, \psi(x)=x^{2}
$$

- Exponential

$$
\phi(x)=\exp (-x), \psi(x)=1-\sqrt{1-x^{2}}
$$

O. Bousquet - Statistical Learning Theory - Lecture 6 


\section{Low noise conditions}

- Relationship can be improved under low noise conditions

- Under Tsybakov's condition with exponent $\alpha$ and constant $c$,

$$
c\left(R(g)-R^{*}\right)^{\alpha} \psi\left(\left(R(g)-R^{*}\right)^{1-\alpha} / 2 c\right) \leq L(g)-L^{*}
$$

- Hinge loss (no improvement)

$$
R(g)-R^{*} \leq L(g)-L^{*}
$$

- Square loss or squared hinge loss

$$
R(g)-R^{*} \leq\left(4 c\left(L(g)-L^{*}\right)\right)^{\frac{1}{2-\alpha}}
$$

O. Bousquet - Statistical Learning Theory - Lecture 6 


\section{Estimation error}

- Recall that Tsybakov condition implies $P f^{2} \leq c(P f)^{\alpha}$ for the relative loss class (with $0-1$ loss)

- What happens for the relative loss class associated to $\phi$ ?

- Two possibilities

* Strictly convex loss (can modify the metric on $\mathbb{R}$ )

$\star$ Piecewise linear

O. Bousquet - Statistical Learning Theory - Lecture 6 


\section{Strictly convex losses}

- Noise behavior controlled by modulus of convexity

- Result

$$
\delta\left(\frac{\sqrt{P f^{2}}}{K}\right) \leq P f / 2
$$

with $K$ Lipschitz constant of $\phi$ and $\delta$ modulus of convexity of $L(g)$ with respect to $\|f-g\|_{L_{2}(P)}$

- Not related to noise exponent 


\section{Piecewise linear losses}

- Noise behavior related to noise exponent

- Result for hinge loss

$$
P f^{2} \leq C P f^{\alpha}
$$

if initial class $\mathcal{G}$ is uniformly bounded 


\section{Estimation error}

- With bounded and Lipschitz loss with convexity exponent $\gamma$, for a convex class $\mathcal{G}$,

$$
L(g)-L\left(g^{*}\right) \leq C\left(\left(r^{*}\right)^{\frac{2}{\gamma}}+\frac{\log \frac{1}{\delta}+\log \log n}{n}\right)
$$

- Under Tsybakov's condition for the hinge loss (and general $\mathcal{G}$ ) $P f^{2} \leq C P f^{\alpha}$

$$
L(g)-L\left(g^{*}\right) \leq C\left(\left(r^{*}\right)^{\frac{1}{2-\alpha}}+\frac{\log \frac{1}{\delta}+\log \log n}{n}\right)
$$

O. Bousquet - Statistical Learning Theory - Lecture 6 


\section{Examples}

\section{Under Tsybakov's condition}

- Hinge loss

$$
R(g)-R^{*} \leq L\left(g^{*}\right)-L^{*}+C\left(\left(r^{*}\right)^{\frac{1}{2-\alpha}}+\frac{\log \frac{1}{\delta}+\log \log n}{n}\right)
$$

- Squared hinge loss or square loss $\delta(x)=c x^{2}, P f^{2} \leq C P f$

$$
R(g)-R^{*} \leq C\left(L\left(g^{*}\right)-L^{*}+C^{\prime}\left(r^{*}+\frac{\log \frac{1}{\delta}+\log \log n}{n}\right)\right)^{\frac{1}{2-\alpha}}
$$

O. Bousquet - Statistical Learning Theory - Lecture 6 


\section{Classification vs Regression losses}

- Consider a classification-calibrated function $\phi$

- It is a classification loss if $L(t)=L^{*}$

- otherwise it is a regression loss

O. Bousquet - Statistical Learning Theory - Lecture 6 


\section{Classification vs Regression losses}

- Square, squared hinge, exponential losses

* Noise enters relationship between risk and loss

$\star$ Modulus of convexity enters in estimation error

- Hinge loss

$\star$ Direct relationship between risk and loss

* Noise enters in estimation error

$\Rightarrow$ Approximation term not affected by noise in second case

$\Rightarrow$ Real value does not bring probability information in second case

O. Bousquet - Statistical Learning Theory - Lecture 6 


\section{Lecture 7}

\section{Regularization}

- Formulation

- Capacity measures

- Computing Rademacher averages

- Applications 


\section{Equivalent problems}

Up to the choice of the regularization parameters, the following problems are equivalent

$$
\begin{gathered}
\min _{f \in \mathcal{F}} L_{n}(f)+\lambda \Omega(f) \\
\min _{f \in \mathcal{F}: L_{n}(f) \leq e} \Omega(f) \\
\min _{f \in \mathcal{F}: \Omega(f) \leq R} L_{n}(f)
\end{gathered}
$$

The solution sets are the same

O. Bousquet - Statistical Learning Theory - Lecture 7 


\section{Comments}

- Computationally, variant of SRM

- variant of model selection by penalization

$\Rightarrow$ one has to choose a regularizer which makes sense

- Need a class that is large enough (for universal consistency)

- but has small balls

O. Bousquet - Statistical Learning Theory - Lecture 7 


\section{Rates}

- To obtain bounds, consider ERM on balls

- Relevant capacity is that of balls

- Real-valued functions, need a generalization of VC dimension, entropy or covering numbers

- Involve scale sensitive capacity (takes into account the value and not only the sign) 


\section{Scale-sensitive capacity}

- Generalization of VC entropy and VC dimension to real-valued functions

- Definition: a set $x_{1}, \ldots, x_{n}$ is shattered by $\mathcal{F}$ (at scale $\varepsilon$ ) if there exists a function $s$ such that for all choices of $\alpha_{i} \in\{-1,1\}$, there exists $f \in \mathcal{F}$

$$
\alpha_{i}\left(f\left(x_{i}\right)-s\left(x_{i}\right)\right) \geq \varepsilon
$$

- The fat-shattering dimension of $\mathcal{F}$ at scale $\varepsilon($ denoted $v c(\mathcal{F}, \varepsilon)$ ) is the maximum cardinality of a shattered set 


\section{Link with covering numbers}

- Like VC dimension, fat-shattering dimension can be used to upper bound covering numbers

- Result

$$
N(\mathcal{F}, t, n) \leq\left(\frac{C_{1}}{t}\right)^{C_{2} v c\left(\mathcal{F}, C_{3} t\right)}
$$

- Note that one can also define data-dependent versions (restriction on the sample)

O. Bousquet - Statistical Learning Theory - Lecture 7 


\section{Link with Rademacher averages (1)}

- Consequence of covering number estimates

$$
\mathbb{R}_{n}(\mathcal{F}) \leq \frac{C_{1}}{\sqrt{n}} \int_{0}^{\infty} \sqrt{v c(\mathcal{F}, t) \log \frac{C_{2}}{t}} d t
$$

- Another link via Gaussian averages (replace Rademacher by Gaussian $\mathrm{N}(0,1)$ variables $)$

$$
\mathcal{G}_{n}(\mathcal{F})=\mathbb{E}_{g}\left[\sup _{f \in \mathcal{F}} \frac{1}{n} \sum_{i=1}^{n} g_{i} f\left(Z_{i}\right)\right]
$$

O. Bousquet - Statistical Learning Theory - Lecture 7 


\section{Link with Rademacher averages (2)}

- Worst case average

$$
\ell_{n}(\mathcal{F})=\sup _{x_{1}, \ldots, x_{n}} \mathbb{E}_{\sigma}\left[\sup _{f \in \mathcal{F}} G_{n} f\right]
$$

- Associated "dimension" $t(\mathcal{F}, \epsilon)=\sup \left\{n \in \mathbb{N}: \ell_{n}(\mathcal{F}) \geq \epsilon\right\}$

- Result (Mendelson \& Vershynin 2003)

$$
v c\left(\mathcal{F}, c^{\prime} \epsilon\right) \leq t(\mathcal{F}, \epsilon) \leq \frac{K}{\epsilon^{2}} v c(\mathcal{F}, c \epsilon)
$$

O. Bousquet - Statistical Learning Theory - Lecture 7 


\section{Rademacher averages and Lipschitz losses}

- What matters is the capacity of $\mathcal{F}$ (loss class)

- If $\phi$ is Lipschitz with constant $M$

- then

$$
\mathcal{R}_{n}(\mathcal{F}) \leq M \mathcal{R}_{n}(\mathcal{G})
$$

- Relates to Rademacher average of the initial class (easier to compute) 


\section{Dualization}

- Consider the problem $\min _{\|g\| \leq R} L_{n}(g)$

- Rademacher of ball

$$
\mathbb{E}_{\sigma}\left[\sup _{\|g\| \leq R} R_{n} g\right]
$$

- Duality

$$
\mathbb{E}_{\sigma}\left[\sup _{\|f\| \leq R} R_{n} f\right]=\frac{R}{n} \mathbb{E}_{\sigma}\left[\left\|\sum_{i=1}^{n} \sigma_{i} \delta_{X_{i}}\right\|^{*}\right]
$$

\|\|$^{*}$ dual norm, $\delta_{X_{i}}$ evaluation at $X_{i}$ (element of the dual under appropriate conditions)

O. Bousquet - Statistical Learning Theory - Lecture 7 


\section{RHKS}

Given a positive definite kernel $k$

- Space of functions: reproducing kernel Hilbert space associated to $k$

- Regularizer: rkhs norm $\|\cdot\|_{k}$

- Properties: Representer theorem

$$
g_{n}=\sum_{i=1}^{n} \alpha_{i} k\left(X_{i}, \cdot\right)
$$

O. Bousquet - Statistical Learning Theory - Lecture 7 


\section{Shattering dimension of hyperplanes}

- Set of functions

$$
\mathcal{G}=\{g(\mathbf{x})=\mathbf{w} \cdot \mathbf{x}:\|\mathbf{w}\|=1\}
$$

- Assume $\|\mathbf{x}\| \leq R$

- Result

$$
v c(\mathcal{G}, \rho) \leq R^{2} / \rho^{2}
$$

O. Bousquet - Statistical Learning Theory - Lecture 7 


\section{Proof Strategy (Gurvits, 1997)}

Assume that $\mathbf{x}_{1}, \ldots, \mathbf{x}_{r}$ are $\rho$-shattered by hyperplanes with $\|\mathbf{w}\|=1$, i.e., for all $y_{1}, \ldots, y_{r} \in\{ \pm 1\}$, there exists a $\mathbf{w}$ such that

$$
y_{i}\left\langle\mathbf{w}, \mathbf{x}_{i}\right\rangle \geq \rho \text { for all } i=1, \ldots, r .
$$

Two steps:

- prove that the more points we want to shatter (2), the larger $\left\|\sum_{i=1}^{r} y_{i} \mathbf{x}_{i}\right\|$ must be

- upper bound the size of $\left\|\sum_{i=1}^{r} y_{i} \mathbf{x}_{i}\right\|$ in terms of $R$

Combining the two tells us how many points we can at most shatter 


\section{Part I}

- Summing (2) yields $\left\langle\mathbf{w},\left(\sum_{i=1}^{r} y_{i} \mathbf{x}_{i}\right)\right\rangle \geq r \rho$

- By Cauchy-Schwarz inequality

$$
\left\langle\mathbf{w},\left(\sum_{i=1}^{r} y_{i} \mathbf{x}_{i}\right)\right\rangle \leq\|\mathbf{w}\|\left\|\sum_{i=1}^{r} y_{i} \mathbf{x}_{i}\right\|=\left\|\sum_{i=1}^{r} y_{i} \mathbf{x}_{i}\right\|
$$

- Combine both:

$$
r \rho \leq\left\|\sum_{i=1}^{r} y_{i} \mathbf{x}_{i}\right\| .
$$

O. Bousquet - Statistical Learning Theory - Lecture 7 


\section{Part II}

Consider labels $y_{i} \in\{ \pm 1\}$, as (Rademacher variables).

$$
\begin{aligned}
\mathbb{E}\left[\left\|\sum_{i=1}^{r} y_{i} \mathbf{x}_{i}\right\|^{2}\right] & =\mathbb{E}\left[\sum_{i, j=1}^{r} y_{i} y_{j}\left\langle\mathbf{x}_{i}, \mathbf{x}_{j}\right\rangle\right] \\
& =\sum_{i=1}^{r} \mathbb{E}\left[\left\langle\mathbf{x}_{i}, \mathbf{x}_{i}\right\rangle\right]+\mathbb{E}\left[\sum_{i \neq j}^{r}\left\langle\mathbf{x}_{i}, \mathbf{x}_{j}\right\rangle\right] \\
& =\sum_{i=1}^{r}\left\|\mathbf{x}_{i}\right\|^{2}
\end{aligned}
$$

O. Bousquet - Statistical Learning Theory - Lecture 7 


\section{Part II, ctd.}

- Since $\left\|\mathbf{x}_{i}\right\| \leq R$, we get $\mathbb{E}\left[\left\|\sum_{i=1}^{r} y_{i} \mathbf{x}_{i}\right\|^{2}\right] \leq r R^{2}$.

- This holds for the expectation over the random choices of the labels, hence there must be at least one set of labels for which it also holds true. Use this set.

- Hence

$$
\left\|\sum_{i=1}^{r} y_{i} \mathbf{x}_{i}\right\|^{2} \leq r R^{2} .
$$

O. Bousquet - Statistical Learning Theory - Lecture 7 


\section{Part I and II Combined}

- Part I: $(r \rho)^{2} \leq\left\|\sum_{i=1}^{r} y_{i} \mathbf{x}_{i}\right\|^{2}$

- Part II: $\left\|\sum_{i=1}^{r} y_{i} \mathbf{x}_{i}\right\|^{2} \leq r R^{2}$

- Hence

$$
r^{2} \rho^{2} \leq r R^{2}
$$

i.e.,

$$
r \leq \frac{R^{2}}{\rho^{2}}
$$

O. Bousquet - Statistical Learning Theory - Lecture 7 


\section{Boosting}

Given a class $\mathcal{H}$ of functions

- Space of functions: linear span of $\mathcal{H}$

- Regularizer: 1-norm of the weights $\|g\|=\inf \left\{\sum\left|\alpha_{i}\right|: g=\right.$ $\left.\sum \alpha_{i} h_{i}\right\}$

- Properties: weight concentrated on the (weighted) margin maximizers

$$
\begin{aligned}
g_{n} & =\sum w_{h} h \\
\sum d_{i} Y_{i} h\left(X_{i}\right) & =\min _{h^{\prime} \in \mathcal{H}} \sum d_{i} Y_{i} h^{\prime}\left(X_{i}\right)
\end{aligned}
$$

O. Bousquet - Statistical Learning Theory - Lecture 7 


\section{Rademacher averages for boosting}

- Function class of interest

$$
\mathcal{G}_{R}=\left\{g \in \operatorname{span} \mathcal{H}:\|g\|_{1} \leq R\right\}
$$

- Result

$$
\mathcal{R}_{n}\left(\mathcal{G}_{R}\right)=R \mathcal{R}_{n}(\mathcal{H})
$$

$\Rightarrow$ Capacity (as measured by global Rademacher averages) not affected by taking linear combinations !

O. Bousquet - Statistical Learning Theory - Lecture 7 


\section{Lecture 8}

\section{SVM}

- Computational aspects

- Capacity Control

- Universality

- Special case of RBF kernel

O. Bousquet - Statistical Learning Theory - Lecture 8 


\section{Formulation (1)}

- Soft margin

$$
\begin{gathered}
\min _{\mathbf{w}, b, \xi} \frac{1}{2}\|\mathbf{w}\|^{2}+C \sum_{i=1}^{m} \xi_{i} \\
y_{i}\left(\left\langle\mathbf{w}, \mathbf{x}_{i}\right\rangle+b\right) \geq 1-\xi_{i} \\
\xi_{i} \geq 0
\end{gathered}
$$

- Convex objective function and convex constraints

- Unique solution

- Efficient procedures to find it

$\rightarrow$ Is it the right criterion?

O. Bousquet - Statistical Learning Theory - Lecture 8 


\section{Formulation (2)}

- Soft margin

$$
\begin{gathered}
\min _{\mathbf{w}, b, \xi} \frac{1}{2}\|\mathbf{w}\|^{2}+C \sum_{i=1}^{m} \xi_{i} \\
y_{i}\left(\left\langle\mathbf{w}, \mathbf{x}_{i}\right\rangle+b\right) \geq 1-\xi_{i}, \quad \xi_{i} \geq 0
\end{gathered}
$$

- Optimal value of $\xi_{i}$$$
\xi_{i}^{*}=\max \left(0,1-y_{i}\left(\left\langle\mathbf{w}, \mathbf{x}_{i}\right\rangle+b\right)\right)
$$

- Substitute above to get

$$
\min _{\mathbf{w}, b} \frac{1}{2}\|\mathbf{w}\|^{2}+C \sum_{i=1}^{m} \max \left(0,1-y_{i}\left(\left\langle\mathbf{w}, \mathbf{x}_{i}\right\rangle+b\right)\right)
$$

O. Bousquet - Statistical Learning Theory - Lecture 8 


\section{Regularization}

General form of regularization problem

$$
\min _{f \in \mathcal{F}} \frac{1}{m} \sum_{i=1}^{n} c\left(y_{i} f\left(x_{i}\right)\right)+\lambda\|f\|^{2}
$$

$\rightarrow$ Capacity control by regularization with convex cost

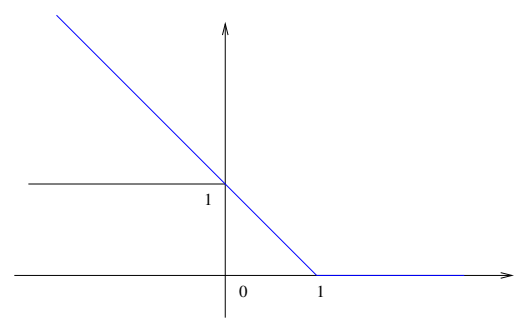

O. Bousquet - Statistical Learning Theory - Lecture 8 


\section{Loss Function}

$$
\phi(Y f(X))=\max (0,1-Y f(X))
$$

- Convex, non-increasing, upper bounds $1_{[Y f(X) \leq 0]}$

- Classification-calibrated

- Classification type $\left(L^{*}=L(t)\right)$

$$
R(g)-R^{*} \leq L(g)-L^{*}
$$

O. Bousquet - Statistical Learning Theory - Lecture 8 


\section{Regularization}

Choosing a kernel corresponds to

- Choose a sequence $\left(a_{k}\right)$

- Set

$$
\|f\|^{2}:=\sum_{k \geq 0} a_{k} \int\left|f^{(k)}\right|^{2} d x
$$

$\Rightarrow$ penalization of high order derivatives (high frequencies)

$\Rightarrow$ enforce smoothness of the solution

O. Bousquet - Statistical Learning Theory - Lecture 8 


\section{Capacity: VC dimension}

- The VC dimension of the set of hyperplanes is $d+1$ in $\mathbb{R}^{d}$.

Dimension of feature space?

$\infty$ for RBF kernel

- $w$ choosen in the span of the data $\left(w=\sum \alpha_{i} y_{i} \mathbf{x}_{i}\right)$

The span of the data has dimension $m$ for RBF kernel $\left(k\left(., x_{i}\right)\right.$ linearly independent)

- The VC bound does not give any information

$$
\sqrt{\frac{h}{m}}=1
$$

$\Rightarrow$ Need to take the margin into account

O. Bousquet - Statistical Learning Theory - Lecture 8 


\section{Capacity: Shattering dimension}

\section{Hyperplanes with Margin}

If $\|x\| \leq R$,

$v c($ hyperplanes with margin $\rho, 1) \leq R^{2} / \rho^{2}$

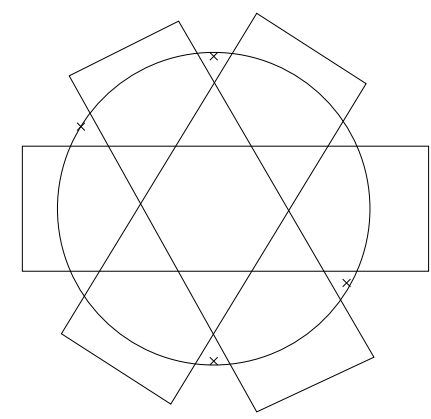

O. Bousquet - Statistical Learning Theory - Lecture 8 


\section{Margin}

- The shattering dimension is related to the margin

- Maximizing the margin means minimizing the shattering dimension

- Small shattering dimension $\Rightarrow$ good control of the risk

$\Rightarrow$ this control is automatic (no need to choose the margin beforehand)

$\Rightarrow$ but requires tuning of regularization parameter

O. Bousquet - Statistical Learning Theory - Lecture 8 


\section{Capacity: Rademacher Averages (1)}

- Consider hyperplanes with $\|w\| \leq M$

- Rademacher average

$$
\frac{M}{n \sqrt{2}} \sqrt{\sum_{i=1}^{n} k\left(x_{i}, x_{i}\right)} \leq \mathcal{R}_{n} \leq \frac{M}{n} \sqrt{\sum_{i=1}^{n} k\left(x_{i}, x_{i}\right)}
$$

- Trace of the Gram matrix

- Notice that $\mathcal{R}_{n} \leq \sqrt{R^{2} /\left(n^{2} \rho^{2}\right)}$ 


\section{Rademacher Averages (2)}

$$
\begin{aligned}
\mathbb{E} & {\left[\sup _{\|w\| \leq M} \frac{1}{n} \sum_{i=1}^{n} \sigma_{i}\left\langle w, \delta_{x_{i}}\right\rangle\right] } \\
& =\mathbb{E}\left[\sup _{\|w\| \leq M}\left\langle w, \frac{1}{n} \sum_{i=1}^{n} \sigma_{i} \delta_{x_{i}}\right\rangle\right] \\
& \leq \mathbb{E}\left[\sup _{\|w\| \leq M}\|w\|\left\|\frac{1}{n} \sum_{i=1}^{n} \sigma_{i} \delta_{x_{i}}\right\|\right] \\
& =\frac{M}{n} \mathbb{E}\left[\sqrt{\left\langle\sum_{i=1}^{n} \sigma_{i} \delta_{x_{i}}, \sum_{i=1}^{n} \sigma_{i} \delta_{x_{i}}\right\rangle}\right]
\end{aligned}
$$

O. Bousquet - Statistical Learning Theory - Lecture 8 


\section{Rademacher Averages (3)}

$$
\begin{aligned}
\frac{M}{n} & \mathbb{E}\left[\sqrt{\left\langle\sum_{i=1}^{n} \sigma_{i} \delta_{x_{i}}, \sum_{i=1}^{n} \sigma_{i} \delta_{x_{i}}\right\rangle}\right] \\
& \leq \frac{M}{n} \sqrt{\mathbb{E}\left[\left\langle\sum_{i=1}^{n} \sigma_{i} \delta_{x_{i}}, \sum_{i=1}^{n} \sigma_{i} \delta_{x_{i}}\right\rangle\right]} \\
& =\frac{M}{n} \sqrt{\mathbb{E}\left[\sum_{i, j} \sigma_{i} \sigma_{j}\left\langle\delta_{x_{i}}, \delta_{x_{j}}\right\rangle\right]} \\
& =\frac{M}{n} \sqrt{\sum_{i=1}^{n} k\left(x_{i}, x_{i}\right)}
\end{aligned}
$$

O. Bousquet - Statistical Learning Theory - Lecture 8 


\section{Improved rates - Noise condition}

- Under Massart's condition $\left(|\eta|>\eta_{0}\right)$, with $\|g\|_{\infty} \leq M$

$$
\mathbb{E}\left[(\phi(Y g(X))-\phi(Y t(X)))^{2}\right] \leq\left(M-1+2 / \eta_{0}\right)\left(L(g)-L^{*}\right) .
$$

$\rightarrow$ If noise is nice, variance linearly related to expectation

$\rightarrow$ Estimation error of order $r^{*}$ (of the class $\mathcal{G}$ ) 


\section{Improved rates - Capacity (1)}

- $r_{n}^{*}$ related to decay of eigenvalues of the Gram matrix

$$
r_{n}^{*} \leq \frac{c}{n} \min _{d \in \mathbb{N}}\left(d+\sqrt{\sum_{j>d} \lambda_{j}}\right)
$$

- Note that $d=0$ gives the trace bound

- $r_{n}^{*}$ always better than the trace bound (equality when $\lambda_{i}$ constant) 


\section{Improved rates - Capacity (2)}

Example: exponential decay

- $\lambda_{i}=e^{-\alpha i}$

- Global Rademacher of order $\frac{1}{\sqrt{n}}$

- $r_{n}^{*}$ of order

$$
\frac{\log n}{n}
$$

O. Bousquet - Statistical Learning Theory - Lecture 8 


\section{Exponent of the margin}

- Estimation error analysis shows that in $\mathcal{G}_{M}=\{g:\|g\| \leq M\}$

$$
R\left(g_{n}\right)-R\left(g^{*}\right) \leq M \ldots
$$

- Wrong power ( $M^{2}$ penalty) is used in the algorithm

- Computationally easier

- But does not give $\lambda$ a dimension-free status

- Using $M$ could improve the cutoff detection 


\section{Kernel}

Why is it good to use kernels?

- Gaussian kernel (RBF)

$$
k(x, y)=e^{-\frac{\|x-y\|^{2}}{2 \sigma^{2}}}
$$

- $\sigma$ is the width of the kernel

$\rightarrow$ What is the geometry of the feature space?

O. Bousquet - Statistical Learning Theory - Lecture 8 


\section{RBF}

\section{Geometry}

- Norms

$$
\|\Phi(x)\|^{2}=\langle\Phi(x), \Phi(x)\rangle=e^{0}=1
$$

$\rightarrow$ sphere of radius 1

- Angles

$$
\cos \left(\Phi(\widehat{x), \Phi}(y))=\left\langle\frac{\Phi(x)}{\|\Phi(x)\|}, \frac{\Phi(y)}{\|\Phi(y)\|}\right\rangle=e^{-\|x-y\|^{2} / 2 \sigma^{2}} \geq 0\right.
$$

$\rightarrow$ Angles less than 90 degrees

- $\Phi(x)=k(x,) \geq$.

$\rightarrow$ positive quadrant 


\section{RBF}

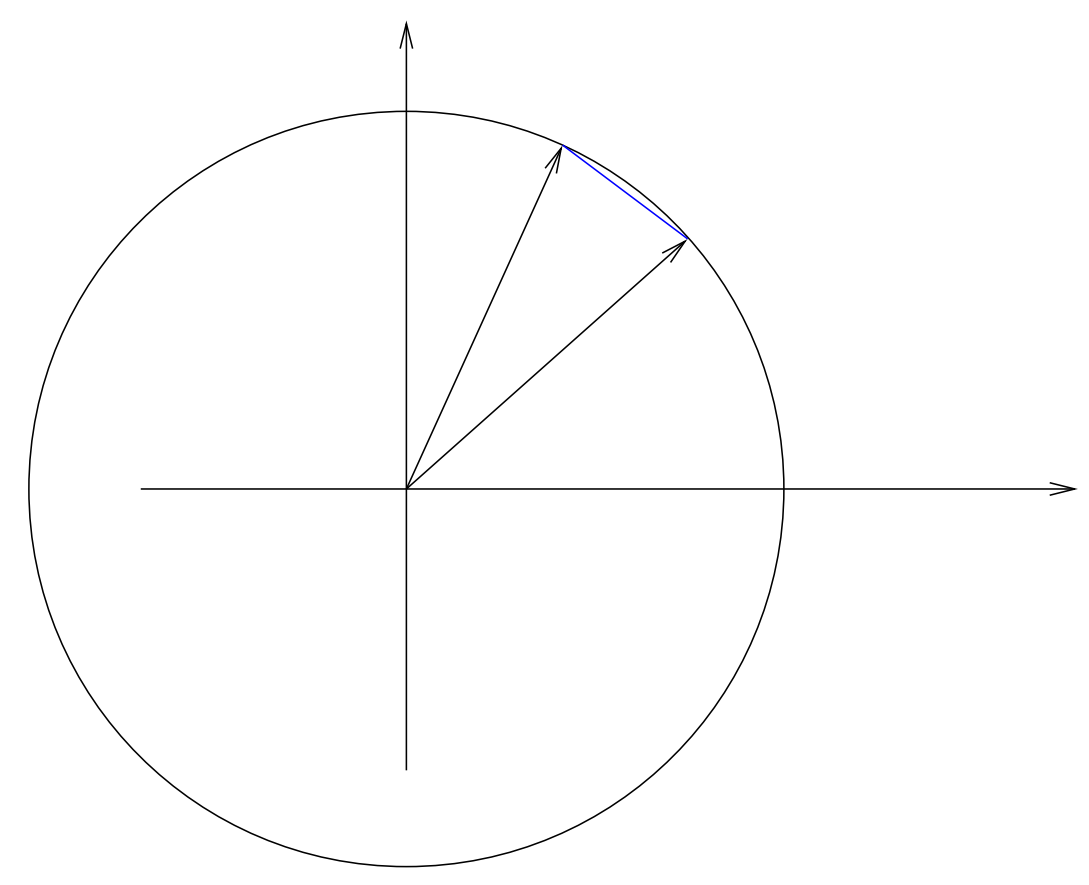

O. Bousquet - Statistical Learning Theory - Lecture 8 


\section{RBF}

\section{Differential Geometry}

- Flat Riemannian metric

$\rightarrow$ 'distance' along the sphere is equal to distance in input space

- Distances are contracted

$\longrightarrow$ 'shortcuts' by getting outside the sphere

O. Bousquet - Statistical Learning Theory - Lecture 8 


\section{RBF}

\section{Geometry of the span}

Ellipsoid

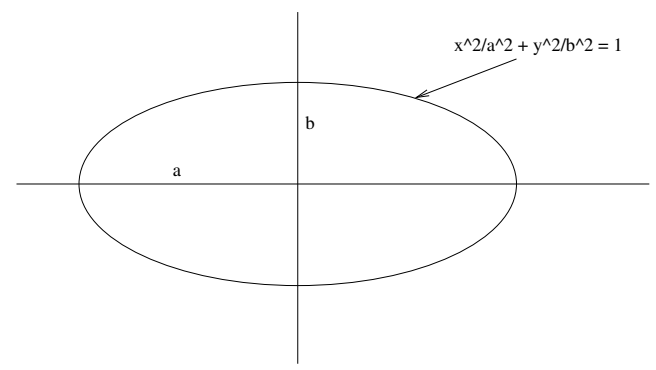

- $K=\left(k\left(x_{i}, x_{j}\right)\right)$ Gram matrix

- Eigenvalues $\lambda_{1}, \ldots, \lambda_{m}$

- Data points mapped to ellispoid with lengths $\sqrt{\lambda_{1}}, \ldots, \sqrt{\lambda_{m}}$

O. Bousquet - Statistical Learning Theory - Lecture 8 


\section{RBF}

\section{Universality}

- Consider the set of functions

$$
\mathcal{H}=\operatorname{span}\{k(x, \cdot): x \in \mathcal{X}\}
$$

- $\mathcal{H}$ is dense in $C(\mathcal{X})$

$\rightarrow$ Any continuous function can be approximated (in the \|\|$_{\infty}$ norm) by functions in $\mathcal{H}$

$\Rightarrow$ with enough data one can construct any function

O. Bousquet - Statistical Learning Theory - Lecture 8 


\section{RBF}

\section{Eigenvalues}

- Exponentially decreasing

- Fourier domain: exponential penalization of derivatives

- Enforces smoothness with respect to the Lebesgue measure in input space

O. Bousquet - Statistical Learning Theory - Lecture 8 


\section{RBF}

\section{Induced Distance and Flexibility}

- $\sigma \rightarrow 0$

1-nearest neighbor in input space

Each point in a separate dimension, everything orthogonal

- $\sigma \rightarrow \infty$

linear classifier in input space

All points very close on the sphere, initial geometry

- Tuning $\sigma$ allows to try all possible intermediate combinations

O. Bousquet - Statistical Learning Theory - Lecture 8 


\section{RBF}

\section{Ideas}

- Works well if the Euclidean distance is good

- Works well if decision boundary is smooth

- Adapt smoothness via $\sigma$

- Universal

O. Bousquet - Statistical Learning Theory - Lecture 8 


\section{Choosing the Kernel}

- Major issue of current research

- Prior knowledge (e.g. invariances, distance)

- Cross-validation (limited to 1-2 parameters)

- Bound (better with convex class)

$\Rightarrow$ Lots of open questions...

O. Bousquet - Statistical Learning Theory - Lecture 8 


\section{Learning Theory: some informal thoughts}

- Need assumptions/restrictions to learn

- Data cannot replace knowledge

- No universal learning (simplicity measure)

- SVM work because of capacity control

- Choice of kernel = choice of prior/ regularizer

- RBF works well if Euclidean distance meaningful

- Knowledge improves performance (e.g. invariances)

O. Bousquet - Statistical Learning Theory - Lecture 8 